\title{
ASYMPTOTICAL DYNAMICS FOR NON-AUTONOMOUS STOCHASTIC EQUATIONS DRIVEN BY A NON-LOCAL INTEGRO-DIFFERENTIAL OPERATOR OF FRACTIONAL TYPE
}

\author{
Wenqiang Zhao \\ Chongqing Technology and Business University, School of Mathematics and Statistics \\ Chongqing 400067, P. R. China; gshzhao@sina.com
}

\begin{abstract}
The purpose of this paper is to investigate the asymptotical dynamics of solutions for a non-autonomous stochastic evolution equations driven by a non-local integro-differential operator $\mathcal{L}_{K}$ defined by

$$
\mathcal{L}_{K} u(x)=P . V . \int_{\mathbf{R}^{N}}(u(x)-u(y)) K(x-y) d y, \quad x \in \mathbf{R}^{N},
$$

where $K: \mathbf{R}^{N} \backslash\{0\} \rightarrow(0,+\infty)$ is the kernel of $\mathcal{L}_{K}$ which satisfies the general fractional-type condition of order $s$. It is showed that the $(2 p-2)$-truncation of solutions on a finite integral interval vanishes if the initial time goes to negative infinite and the eigenvalue of $\mathcal{L}_{K}$ is large enough. By means of this truncation estimate and the spectrum splitting technique, the flattening condition of solutions is proved in the fractional-type Sobolev space $X_{0}^{s}$, under a weak assumption on the non-autonomous term. Then, the regular dynamics of the cocycle associated with this problem are demonstrated, namely, that the pullback attractor established in $L^{2}(\mathcal{O})$ is actually compact, measurable and attracting in the fractional-type space $X_{0}^{s}$ for any $s \in(0,1)$ and $N>2 s$. As a typical example, we derive the random dynamics for the problem driven by the fractional Laplacian $(-\Delta)^{s}$.
\end{abstract}

\section{Introduction}

In recent years, a huge volume of literature focus on the problems involving the fractional and non-local operators, since such type of operators arise from a diverse scientific fields, such as fluid mechanics, biology, finance, optimization, quantum theory and etc. For a survey of this topic, the reader is referred to [7, 20].

Let $\mathcal{O} \subset \mathbf{R}^{N}$ be an open and bounded domain with Lipschitz boundary. In this article, we are interested in the asymptotical dynamics (in the form of pullback attractor) of solutions of the non-autonomous stochastic general integro-differential equation on $\mathcal{O}$ :

$$
\frac{d u}{d t}+\mathcal{L}_{K} u=F(t, x, u)+g(t, x)+\phi(x) \dot{W}(t), \quad t>\tau, x \in \mathcal{O},
$$

with the homogeneous Dirichlet boundary condition

$$
u(t, x)=0, \quad t>\tau, x \in \mathbf{R}^{N} \backslash \mathcal{O},
$$

and the initial condition

$$
u(\tau, x)=u_{\tau}, \quad x \in \mathcal{O},
$$

https://doi.org/10.5186/aasfm.2019.4414

2010 Mathematics Subject Classification: Primary 35R60, 35B40, 35B41, 35B65.

Key words: Non-autonomous integro-differential equation, non-local operator, fractional Laplacian, pullback attractor, additive noise, flattening condition. 
where $\tau \in \mathbf{R}, g \in L_{l o c}^{2}\left(\mathbf{R}, L^{2}(\mathcal{O})\right)$. Throughout this paper, $W(t)$ is a two-sided realvalued Brownian motion defined on a probability space, $F: \mathbf{R} \times \mathcal{O} \times \mathbf{R} \rightarrow \mathbf{R}$ is a continuous function, $\phi$ is a given function on $\mathbf{R}^{N}$ satisfying some conditions as specified in the latter section, and $\mathcal{L}_{K}$ is the non-local integro-differential operator of order $s$ suggested in [24] and defined as follows:

$$
\begin{aligned}
\mathcal{L}_{K} u(x) & =P . V \cdot \int_{\mathbf{R}^{N}}(u(x)-u(y)) K(x-y) d y \\
& =\lim _{\varepsilon \searrow 0} \int_{\mathbf{R}^{N} \backslash \mathcal{B}_{\varepsilon}(x)}(u(x)-u(y)) K(x-y) d y, \quad x \in \mathbf{R}^{N},
\end{aligned}
$$

where $\mathcal{B}_{\varepsilon}(x)$ is a ball in $\mathbf{R}^{N}$ centred at $x$ with radius $\varepsilon, P . V$. means the principal value of the integral, and the kernel $K: \mathbf{R}^{N} \backslash\{0\} \rightarrow(0,+\infty)$ is a measurable function satisfying

$$
\begin{aligned}
& m K \in L^{1}\left(\mathbf{R}^{N}\right), \text { where } m(x)=\min \left\{1, x^{2}\right\}, \\
& K(x)=K(-x) \text { for any } x \in \mathbf{R}^{N} \backslash\{0\},
\end{aligned}
$$

and there exists $\theta>0$ such that

$$
K(x) \geqslant \theta|x|^{-(N+2 s)} \text { for any } x \in \mathbf{R}^{N} \backslash\{0\},
$$

where $s \in(0,1)$ and $N>2 s$. Condition (1.6) is assumed for sake of simplicity and it can be easily removed (cf. [26]).

We emphasize that the Dirichlet datum $u=0$ in (1.2) is given in $\mathbf{R}^{N} \backslash \mathcal{O}$ instead of simply $u=0$ on the boundary $\partial \mathcal{O}$, which is consistent with the non-local character of the integro-differential operator $\mathcal{L}_{K}$, see the interpretation as in [24, 26, 25].

A typical example for $K$ is the singular kernel $K(x)=|x|^{-(N+2 s)}$. In this case, $\mathcal{L}_{K} u=(-\Delta)^{s} u$ is called the fractional Lapalcian operator. Up to a normalization factor, this operator is defined as

$$
(-\Delta)^{s} u(x)=P . V . \int_{\mathbf{R}^{N}} \frac{u(x)-u(y)}{|x-y|^{n+2 s}} d y=\lim _{\varepsilon \searrow 0} \int_{\mathbf{R}^{N} \backslash \mathcal{B}_{\varepsilon}(x)} \frac{u(x)-u(y)}{|x-y|^{n+2 s}} d y, x \in \mathbf{R}^{N}
$$

Then, the general no-local integro-differential equation (1.1) is reduced to the following usual fractional Laplacian equation:

$$
\frac{d u}{d t}+(-\Delta)^{s} u=F(t, x, u)+g(t, x)+\phi(x) \dot{W}(t),
$$

where $s \in(0,1), N>2 s$.

We can list a large volume of literature about the equations involving such fractional Lapalcian, see e.g. $[9,12,20,21,22,35]$ and the references therein, where the existence of solutions was extensively investigated. However, so far as we know, the asymptotic behavior of solutions for evolution equations with non-local fractional operator are not well studied. Even in the random case, there are only a few publications $[13,17,19,18,29]$ in this aspect, where the authors discussed the existence of random attractor for stochastic fractional reaction-diffusion equations like (1.9) and the fractional complex Ginzburg-Landau equations, respectively. To the best of our knowledge, there is no literature to discuss the long-time dynamical behavior for the stochastic model involving the general non-local operator defined by (1.4). Just as mentioned in [26], it is quite interesting to consider more general operators than the fractional Laplacian, since in the applications, other type of non-local operators naturally arise. 
In this paper, we investigate the asymptotical dynamics and prove that the nonautonomous general model (1.1)-(1.3) has a unique pullback attractor in $L^{2}(\mathcal{O})$ for all $s \in(0,1)$ and $N>2 s$, see Theorem 4.5. We show that the $(2 p-2)$-truncation of solutions on a finite integral interval vanishes if the initial time goes to negative infinite and the eigenvalue of $\mathcal{L}_{K}$ is large enough. The spectrum of the non-local operator $\mathcal{L}_{K}$ is employed by a spectrum splitting approach to obtain the flattening condition of solutions. Then the asymptotically regular dynamics of the model (1.1)(1.3) is demonstrated by a pullback attractor derived in a fractional Sobolev space $X_{0}^{s}$ for all $s \in(0,1)$ and $N>2 s$, see Theorem 5.7. As a typical example, we derive the random dynamics of the fractional reaction-diffusion equations driven by the fractional Laplacian $(-\Delta)^{s}$. Thus the results presented here are new even in the fractional Laplacian case.

We remark that a non-local integro-differential equation containing a kernel different from (1.5) and (1.7) was considered in [8], where the symbolic dynamics was fist studied in a fractional operator setting.

\section{Preliminary results and notations}

In this paper, we consider the general integro-differential equation (1.1). For this, the usual fractional Sobolev space $H^{s}\left(\mathbf{R}^{N}\right)$ or $H^{s}(\mathcal{O})$ (cf. [7]) is not enough. We will work in a functional analytical setting. To this purpose, we present some preliminary results and the fractional-type Sobolev spaces related to the kernel $K($.$) , which will$ be used in the paper.

2.1. The fractional-type Sobolev space with kernel $\boldsymbol{K}($.$) . Let s \in(0,1)$. Define

$$
2_{s}^{*}=\frac{2 N}{N-2 s}, \quad \text { if } N>2 s
$$

which plays the role of a fractional critical Sobolev exponent (cf. [7]). It is clear that $2<2_{s}^{*}=\frac{2 N}{N-2 s}<\frac{2 N}{N-2}=2^{*}$, where the later is the integer critical Sobolev exponent.

Let $X^{s}$ be the linear space of Lebesgue measurable functions form $\mathbf{R}^{N}$ to $\mathbf{R}$ such that the restriction to $\mathcal{O}$ of any function $u$ in $X^{s}$ belongs to $L^{2}(\mathcal{O})$ and

$$
\int_{Q}|u(x)-u(y)|^{2} K(x-y) d x d y<+\infty
$$

where $Q=\mathbf{R}^{2 N} \backslash \mathcal{O}^{c} \times \mathcal{O}^{c}$ with $\mathcal{O}^{c}=\mathbf{R}^{N} \backslash \mathcal{O}$. We note that $Q \supsetneqq \mathcal{O} \times \mathcal{O}$ in general. The space $X^{s}$ is equipped with the norm defined as

$$
\|u\|_{X^{s}}^{2} \triangleq\|u\|_{L^{2}(\mathcal{O})}^{2}+\int_{Q}|u(x)-u(y)|^{2} K(x-y) d x d y .
$$

It is easy to say that $\|\cdot\|_{X^{s}}$ defined by (2.3) is a norm on $X^{s}$ (see [24]) which is equivalent to the following version

$$
\|u\|_{X^{s}} \triangleq\|u\|_{L^{2}(\mathcal{O})}+\left(\int_{Q}|u(x)-u(y)|^{2} K(x-y) d x d y\right)^{\frac{1}{2}} .
$$

Define

$$
X_{0}^{s} \triangleq\left\{u \in X^{s}: u=0 \text { a.e. in } \mathbf{R}^{N} \backslash \mathcal{O}\right\} .
$$

In terms of the condition (1.5), we have $C_{0}^{2}(\mathcal{O}) \subset X_{0}^{s}$ (cf. [20, Lemma 1.20]) and consequently $X_{0}^{s}$ and $X^{s}$ are nonempty. The space $X_{0}^{s}$ is equipped with the norm 
defined as

$$
\begin{aligned}
\|u\|_{X_{0}^{s}}^{2} & \triangleq \int_{Q}|u(x)-u(y)|^{2} K(x-y) d x d y \\
& =\int_{\mathbf{R}^{2 N}}|u(x)-u(y)|^{2} K(x-y) d x d y, \quad u \in X_{0}^{s} .
\end{aligned}
$$

For $u \in X_{0}^{s}$, the integral on the state space $Q$ can be extended to $\mathbf{R}^{2 N}$ as in $[25$, Lemma $5, \mathrm{~b})]$. Moreover, $\left(X_{0}^{s},\|\cdot\|_{X_{0}^{s}}\right)$ is a Hilbert space with the inner product

$$
(u, v)_{X_{0}^{s}} \triangleq\left(\mathcal{L}_{K} u, v\right) \triangleq \int_{Q}(u(x)-u(y))(v(x)-v(y)) K(x-y) d x d y,
$$

and the norm given by (2.5) is equivalent to the usual one defined by (2.3) (cf. [24, Lemma 7]). We also give the usual fractional Sobolev space $H^{s}(\mathcal{O})$ endowed with the so-called Gagliardo norm

$$
\|u\|_{H^{s}(\mathcal{O})}^{2} \triangleq\|u\|_{L^{2}(\mathcal{O})}^{2}+\int_{\mathcal{O} \times \mathcal{O}} \frac{|u(x)-u(y)|^{2}}{\mid\left(x-\left.y\right|^{N+2 s}\right.} d x d y .
$$

For further detailed information on the fractional Sobolev spaces $H^{s}(\mathcal{O})$ and $H^{s}\left(\mathbf{R}^{N}\right)$ for $s \in(0,1)$, the reader is referred to [7] and the references therein.

We remark that, even in the model case in which $k(x)=|x|^{-(N+2 s)}$, the norms in (2.3) and (2.7) are not identical nor equivalent, because $\mathcal{O} \times \mathcal{O}$ is strictly contained in $Q$. This makes the usual fractional Sobolev space approach not sufficient for studying the problem (1.1)-(1.3), especially for the regularity of the solutions [24]. For this, we need the following non-local functional analytic results which formulates the embedding of the space $X_{0}^{s}$ into the usual Lebesgue spaces. They are adapted from Lemma 7-9 in [26].

Lemma 2.1. [26] Let $s \in(0,1), N>2 s, \mathcal{O}$ be an open and bounded domain of $\mathbf{R}^{N}$ and $K: \mathbf{R}^{N} \backslash\{0\} \rightarrow(0, \infty)$ be a measurable function satisfying the conditions (1.5)-(1.7). Then the following assertions hold true:

(i) $X_{0}^{s} \subset H^{s}\left(\mathbf{R}^{N}\right)$ and in addition, there exist a positive constant $c(\theta)$ depending on $\vartheta$ (which is given in (1.7) and $c(\theta, N, s, \mathcal{O})$ depending on $\theta, N, s$ and $\mathcal{O}$ such that for any $u \in X_{0}^{s}$,

$$
\|u\|_{H^{s}(\mathcal{O})} \leqslant\|u\|_{H^{s}\left(\mathbf{R}^{N}\right)} \leqslant c(\theta)\|u\|_{X^{s}} \leqslant c(\theta, N, s, \mathcal{O})\|u\|_{X_{0}^{s}} ;
$$

(ii) Let $k(x)=|x|^{-(N+2 s)}$. Then $X_{0}^{s}=\left\{u \in H^{s}\left(\mathbf{R}^{N}\right): u=0\right.$ a.e. in $\left.\mathbf{R}^{N} \backslash \mathcal{O}\right\}$;

(iii) The embedding $X_{0}^{s} \hookrightarrow L^{q}(\mathcal{O})$ is continuous for any $q \in\left[2,2_{s}^{*}\right]$;

(iv) If $\mathcal{O}$ has a Lipschitz boundary, then the embedding $X_{0}^{s} \hookrightarrow L^{q}(\mathcal{O})$ is compact whenever $q \in\left[2,2_{s}^{*}\right)$.

Remark 1. The compact embedding is closely related to the regularity of the boundary of state space $\mathcal{O}$. As in [26], the Lipschitz boundary in Lemma 2.1 (iv) can be weakened to the continuous boundary, based on [10, Theorem 6]. A simple example of domain with continuous boundary is the ball in $\mathbf{R}^{N}$ and its complement.

2.2. The eigenvalues and eigenfunctions of the non-local operator $\mathcal{L}_{K}$. For our purpose, we need to consider the following eigenvalue problem

$$
\begin{cases}\mathcal{L}_{K}=\lambda u, & \text { in } \mathcal{O} ; \\ u=0, & \text { in } \mathbf{R}^{N} \backslash \mathcal{O}\end{cases}
$$


where $s \in(0,1), N>2 s, \mathcal{O}$ is an open and bounded domain of $\mathbf{R}^{N}$ and $K$ satisfies $(1.5)-1.7)$. More precisely, the problem (2.8) is understood in the weak framework, which is rephrased as the following eigenvalue problem: Finding $u \in X_{0}^{s}$, such that

$$
\int_{\mathbf{R}^{2 N}}\left(u(x)-u(y)(h(x)-h(y)) K(x-y) d x d y=\lambda \int_{\mathcal{O}} u(x) h(x) d x, \quad \forall h \in X_{0}^{s}\right.
$$

The following results, which are adapted from [25], are concerning with the eigenvalue and the corresponding eigenfunction of the problem (2.8) (in fact, of its weak version (2.9)).

Lemma 2.2. ([25]) Let $s \in(0,1), N>2 s, \mathcal{O}$ be an open and bounded domain of $\mathbf{R}^{N}$ and $K: \mathbf{R}^{N} \backslash\{0\} \rightarrow(0, \infty)$ be a measurable function satisfying the conditions (1.5)-(1.7). Then the following assertions hold true:

(i) The first eigenvalue can be characterized as follows

$$
\lambda_{1}=\min _{u \in X_{0}^{s} \backslash\{0\}} \frac{\int_{\mathbf{R}^{2 N}}|u(x)-u(y)|^{2} K(x-y) d x d y}{\int_{\mathcal{O}}|u(x)|^{2} d x} .
$$

Moreover, there exists a non-negative eigenfunction $e_{1} \in X_{0}^{s}$ corresponding to $\lambda_{1}$ with $\left\|e_{1}\right\|_{L^{2}(\mathcal{O})}=1$, such that

$$
\lambda_{1}=\int_{\mathbf{R}^{2 N}}\left|e_{1}(x)-e_{1}(y)\right|^{2} K(x-y) d x d y .
$$

(ii) The problem (2.9) has a family of eigenfunctions $\left\{e_{j}\right\}_{j \in \mathbf{N}}$ such that $\left\{e_{j}\right\}_{j \in \mathbf{N}}$ is an orthonormal basis of $L^{2}(\mathcal{O})$ and an orthonormal basis of $X_{0}^{s}$, and the corresponding eigenvalues $\left\{\lambda_{j}\right\}_{j \in \mathbf{N}}$ satisfies

$$
0<\lambda_{1} \leqslant \lambda_{2} \leqslant \ldots \leqslant \lambda_{j} \rightarrow \infty, \quad \text { as } j \rightarrow \infty .
$$

Moreover, each eigenvalue $\lambda_{j}$ has finite multiplicity.

Moreover, for any $j \in \mathbf{N}$, the eigenvalues can be characterized as follows:

$$
\lambda_{k+1}=\min _{u \in \mathbf{P}_{k+1} \backslash\{0\}} \frac{\int_{\mathbf{R}^{2 N}}|u(x)-u(y)|^{2} K(x-y) d x d y}{\int_{\mathcal{O}}|u(x)|^{2} d x},
$$

where $\mathbf{P}_{k+1}=\left\{u \in X_{0}^{s}:\left\langle u, e_{j}\right\rangle_{X_{0}^{s}}=0, \forall j=1,2, \ldots, k\right\}$. In addition, for any $k \in \mathbf{N}$, there exists a non-negative eigenfunction $e_{k+1} \in \mathbf{P}_{k+1}$ corresponding to the eigenvalue $\lambda_{k+1}$ with $\left\|e_{k+1}\right\|_{L^{2}(\mathcal{O})}=1$, such that

$$
\lambda_{k+1}=\int_{\mathbf{R}^{2 N}}\left|e_{k+1}(x)-e_{k+1}(y)\right|^{2} K(x-y) d x d y .
$$

Throughout this paper, we assume that $s \in(0,1)$ and $N>2 s$.

2.3. Pullback attractor for random cocycle. In this section, we present the notion of pullback attractor and its existence result for the random cocycle. The reader is referred to $[1,3,5,4,23,28,27]$ for more details.

Let $\left(X, d_{X}\right)$ be a Polish spaces (completely separable metric space) with the $\sigma$ algebras $\mathcal{B}(X)$ of Borel subsets. Let $(\Omega, \mathcal{F}, P)$ be a probability space on which there is a $(\mathcal{B}(\mathbf{R}) \times \mathcal{F}, \mathcal{F})$-measurable mapping $\vartheta_{t}: \mathbf{R} \times \Omega \rightarrow \Omega$ such that $\vartheta_{0}=I, \vartheta_{t+\tau}=\vartheta_{t} \circ \vartheta_{\tau}$ and $\vartheta_{t} P=P$ for all $t, \tau \in \mathbf{R}$. The quadruple $\left(\Omega, \mathcal{F}, P,\left\{\vartheta_{t}\right\}_{t \in \mathbf{R}}\right)$ is called a metric (or measurable) dynamical system (briefly, MDS $\vartheta$ ).

Let $\mathbf{R}^{+}=\{t \in \mathbf{R}: t \geqslant 0\}$ and $2^{X}$ be the collection of all subsets of $X$. 
Definition 2.3. Let $D: \mathbf{R} \times \Omega \rightarrow 2^{X} \backslash \emptyset ; D:(\tau, \omega) \rightarrow D(\tau, \omega) \in 2^{X}$ be a setvalued mapping. We say $D:(\tau, \omega) \rightarrow D(\tau, \omega)$ is measurable with respect to $\mathcal{F}$ (briefly, measurable) in $X$ if for every fixed $x \in X$ and $\tau \in \mathbf{R}$, the mapping

$$
\omega \rightarrow d_{X}(x, D(\tau, \omega))=\inf _{z \in D(\tau, \omega)} d_{X}(x, z)
$$

is $(\mathcal{F}, \mathcal{B}(\mathbf{R}))$-measurable. If $D$ is measurable, then the family of its images $D=$ $\{D(\tau, \omega): \tau \in \mathbf{R}, \omega \in \Omega\}$ is also called a random set. If for every fixed $\tau \in \mathbf{R}$ and $\omega \in \Omega$, the image $D(\tau, \omega)$ is closed (resp. compact) in $X$, then the family $D=\{D(\tau, \omega): \tau \in \mathbf{R}, \omega \in \Omega\}$ is called a closed (resp. compact) random set in $X$.

Definition 2.4. A family of single-valued mappings $\varphi: \mathbf{R}^{+} \times \mathbf{R} \times \Omega \times X \rightarrow$ $X,(t, \tau, \omega, x) \mapsto \varphi(t, q, \omega, x)$ is called a random cocycle on $X$ over an MDS $\vartheta$ if for all $s, t \in \mathbf{R}^{+}, \tau \in \mathbf{R}$ and $\omega \in \Omega$, the following statements are satisfied:

- $\varphi(., \tau, .,):. \mathbf{R}^{+} \times \Omega \times X \rightarrow X$ is $\left(\mathcal{B}\left(\mathbf{R}^{+}\right) \times \mathcal{F} \times \mathcal{B}(X), \mathcal{B}(X)\right)$-measurable;

- $\varphi(0, \tau, \omega,$.$) is the identity on \mathrm{X}$;

- $\varphi(t+s, \tau, \omega,)=.\varphi\left(t, \tau+s, \vartheta_{s} \omega, \varphi(s, \tau, \omega,).\right)$.

A random cocycle $\varphi$ is said to be continuous in $X$ if the mapping $\varphi(t, \tau, \omega,):. X \rightarrow X$ is continuous for each $t \in \mathbf{R}^{+}, \tau \in \mathbf{R}$ and $\omega \in \Omega$.

Let $\mathfrak{D}$ a collection of some families of nonempty subsets of $X$, parameterized by the time $\tau$ and the sample $\omega$ such that $\mathfrak{D}=\left\{D=\left\{\emptyset \neq D(\tau, \omega) \in 2^{X}: \tau \in \mathbf{R}, \omega \in\right.\right.$ $\Omega\}$.

Definition 2.5. Let $\mathcal{K}=\{\mathcal{K}(\tau, \omega): \tau \in \mathbf{R}, \omega \in \Omega\} \in \mathfrak{D}$. Then $\mathcal{K}$ is called a $\mathfrak{D}$ pullback absorbing set of the cocycle $\varphi$ over an MDS $\vartheta$ in $X$ if for every $\tau \in \mathbf{R}, \omega \in \Omega$ and $D \in \mathfrak{D}$, there exists an absorbing time $T=T(\tau, \omega, D)>0$ such that

$$
\varphi\left(t, \tau-t, \vartheta_{-t} \omega, D\left(\tau-t, \vartheta_{-t} \omega\right)\right) \subseteq \mathcal{K}(\tau, \omega) \text { for all } t \geqslant T \text {. }
$$

Furthermore, if $\mathcal{K}$ is a random set, then $\mathcal{K}$ is called a $\mathfrak{D}$-pullback random absorbing set in $X$.

Definition 2.6. A family of sets $\mathcal{A}=\{\mathcal{A}(\tau, \omega): \tau \in \mathbf{R}, \omega \in \Omega\} \in \mathfrak{D}$ is called a $\mathfrak{D}$-pullback attractor for the cocycle $\varphi$ over an MDS $\vartheta$ in $X$ if the next three statements hold:

- $\mathcal{A}$ is a compact random set in $X$;

- $\mathcal{A}$ is invariant, that is, for every $\tau \in \mathbf{R}$ and $\omega \in \Omega, \varphi(t, \tau, \omega, \mathcal{A}(\tau, \omega))=$ $\mathcal{A}\left(\tau+t, \vartheta_{t} \omega\right), \forall t \geqslant 0$

- $\mathcal{A}$ is attracting in $X$, namely, for every $\tau \in \mathbf{R}$ and $\omega \in \Omega$ and $D \in \mathfrak{D}$,

$$
\lim _{t \rightarrow \infty} \operatorname{dist}\left(\varphi\left(t, \tau-t, \vartheta_{-t} \omega, D\left(\tau-t, \vartheta_{-t} \omega\right)\right), \mathcal{A}(\tau, \omega)\right)=0
$$

where dist is the Hausdorff semi-metric in $2^{X}$ with $\operatorname{dist}(A, B)=\sup _{x \in A} \inf _{y \in B}$ $d(x, y)$ for $A, B \in 2^{X}$.

If in addition, $\varphi(t, \tau, \omega,$.$) maps X$ into $Y(Y$ is another Polish space) for every $t>$ $0, \tau \in \mathbf{R}$ and $\omega \in \Omega$, and the following conditions are satisfied:

- $\mathcal{A}$ is a compact random set in $Y$;

- $\mathcal{A}$ is attracting in $Y$, namely, for every $\tau \in \mathbf{R}$ and $\omega \in \Omega$ and $D \in \mathfrak{D}$,

$$
\lim _{t \rightarrow \infty} \operatorname{dist}_{Y}\left(\varphi\left(t, \tau-t, \vartheta_{-t} \omega, D\left(\tau-t, \vartheta_{-t} \omega\right)\right), \mathcal{A}(\tau, \omega)\right)=0
$$

then the family $\mathcal{A}$ is called a $\mathfrak{D}$-pullback $(X, Y)$ attractor (briefly,a $\mathfrak{D}$-pullback attractor in $Y$ ). 
We note that the attracting in Definition 2.6 is called pullback attraction, which implies the forward attraction in probability:

$$
\lim _{t \rightarrow \infty} \operatorname{dist}\left(\varphi(t, \tau, \omega, D(\tau, \omega)), \mathcal{A}\left(\tau+t, \vartheta_{t} \omega\right)\right)=0 .
$$

As for the attractor with forward attraction of point-wise convergence, the reader is referred to $[6,15,16]$.

Definition 2.7. The cocycle $\varphi$ over an $\operatorname{MDS} \vartheta$ is said to be $\mathfrak{D}$-pullback asymptotically compact in $X$ if for every $\tau \in \mathbf{R}, \omega \in \Omega$, the sequence

$$
\left\{\varphi\left(t_{n}, \tau-t_{n}, \vartheta_{-t_{n}} \omega, x_{n}\right)\right\}_{n=1}^{\infty} \text { is precompact in } X,
$$

whenever $t_{n} \rightarrow \infty$ and $x_{n} \in D\left(\tau-t_{n}, \vartheta_{-t_{n}} \omega\right)$ with $D \in \mathfrak{D}$.

If in addition, $\varphi(t, \tau, \omega,$.$) maps X$ into $Y$ for every $t>0, \tau \in \mathbf{R}$ and $\omega \in \Omega$, and the sequence

$$
\left\{\varphi\left(t_{n}, \tau-t_{n}, \vartheta_{-t_{n}} \omega, x_{n}\right)\right\}_{n=1}^{\infty} \text { is precompact in } Y,
$$

whenever $t_{n} \rightarrow \infty$ and $x_{n} \in D\left(\tau-t_{n}, \vartheta_{-t_{n}} \omega\right)$ with $D \in \mathfrak{D}$, then $\varphi$ is called $\mathfrak{D}$-pullback asymptotically compact from $X$ to $Y$.

The following existence result for a pullback attractor for a continuous random cocycle can be founded in $[28,27]$. For the existence of pullback attractor in the regular space, we may refer to [34]. We first recall that the a collection $\mathfrak{D}$ of some families of non-empty subsets of $X$ is called inclusion closed if whenever $D=\{D(\tau, \omega): \tau \in \mathbf{R}, \omega \in \Omega\} \in \mathfrak{D}$ with $D^{\prime}(\tau, \omega) \subset D(\tau, \omega)$ for each $\tau \in \mathbf{R}$ and $\omega \in \Omega$, then the family $=D^{\prime}=\left\{D^{\prime}(\tau, \omega): \tau \in \mathbf{R}, \omega \in \Omega\right\} \in \mathfrak{D}$. Note that the inclusion closed collection $\mathfrak{D}$ is also called for short an IC system which was first introduced in [11].

Theorem 2.8. [34] Suppose $\varphi$ is a continuous random cocycle on $X$ over an $\operatorname{MDS} \vartheta$. Let the collection $\mathfrak{D}$ be inclusion closed and $Y$ be another Polish space. Assume that

(i) $\varphi$ has a closed $\mathfrak{D}$-pullback random absorbing set $\mathcal{K}=\{\mathcal{K}(\tau, \omega): \tau \in \mathbf{R}, \omega \in$ $\Omega\} \in \mathfrak{D}$ in $X$;

(ii) $\varphi$ is $\mathfrak{D}$-pullback asymptotically compact in $X$.

Then the random cocycle $\varphi$ admits a unique $\mathfrak{D}$-pullback attractor $\mathcal{A}=\{\mathcal{A}(\tau, \omega): \tau \in$ $\mathbf{R}, \omega \in \Omega\} \in \mathfrak{D}$, where

$$
\mathcal{A}(\tau, \omega)=\bigcap_{s \geqslant 0}{\overline{\bigcup_{t \geqslant s} \varphi\left(t, \tau-t, \vartheta_{-t} \omega, \mathcal{K}\left(\tau-t, \vartheta_{-t} \omega\right)\right)}}^{X} .
$$

If in addition, $\varphi(t, \tau, \omega,$.$) maps X$ into $Y$ for every $t>0, \tau \in \mathbf{R}$ and $\omega \in \Omega$, and $\varphi$ is $\mathfrak{D}$-pullback asymptotically compact from $X$ to $Y$, then the family $\mathcal{A}$ is also a $\mathcal{D}$-pullback attractor in $Y$.

If the target space is a uniformly convex Banach space, such as the Sobolev spaces, the asymptotical compactness is equivalent to the flattening condition. Precisely we have

Theorem 2.9. [14] Suppose that $X$ is a uniformly convex Banach space, then $\varphi$ is $\mathfrak{D}$-pullback asymptotically compact $X$ if and only if $\varphi$ satisfies the $\mathfrak{D}$-flattening condition: For every $\tau \in \mathbf{R}, \omega \in \Omega$ and $D \in \mathfrak{D}$, there exist $T=T(\tau, \omega, D, \varepsilon)>0$ and a finite dimensional space $X_{1}$ of $X$ such that for a bounded projector $P: X \rightarrow X_{1}$,

$$
P\left(\varphi\left(t, \tau-t, \vartheta_{-t} \omega, D\left(\tau-t, \vartheta_{-t} \omega\right)\right) \text { is bounded in } X\right.
$$


and

$$
\|(I-P)\left(\varphi\left(t, \tau-t, \vartheta_{-t} \omega, D\left(\tau-t, \vartheta_{-t} \omega\right)\right) \|_{X} \leqslant \varepsilon\right.
$$

for all $t \geqslant T$.

\section{Random cocycle for the stochastic non-local integro-differential equation}

In what follows, we consider the classic Wiener probability space $(\Omega, \mathcal{F}, P)$, where $\Omega=\{\omega \in C(\mathbf{R}, \mathbf{R}): \omega(0)=0\}$ with the compact-open topology, $\mathcal{F}$ is its Borel $\sigma$ algebra, $P$ is the Wiener measure on $(\Omega, \mathcal{F})$. Then the Brownian motion is identified as $W(t)=W(t, \omega)=\omega(t)$. Define a mapping $\vartheta_{t}: \Omega \times \mathbf{R} \rightarrow \Omega$ such that $\vartheta_{t} \omega()=$. $\omega(t+)-.\omega(t), \forall t \in \mathbf{R}$. Then the quadruple form $\left(\Omega, \mathcal{F}, P,\left\{\vartheta_{t}\right\}_{t \in \mathbf{R}}\right)$ is a metric dynamical system (cf. [1]).

In this section, we will show the existence of random cocycle for the non-autonomous non-local equations on $\mathcal{O}$, driven by an additive noise,

$$
\frac{d u}{d t}+\mathcal{L}_{K} u=F(t, x, u)+g(t, x)+\phi(x) \dot{W}(t), \quad t>\tau, \tau \in \mathbf{R},
$$

with the homogeneous boundary condition

$$
u(t, x)=0, \quad t>\tau, x \in \mathbf{R}^{N} \backslash \mathcal{O},
$$

and the initial condition

$$
u(\tau, x)=u_{\tau}, \quad x \in \mathcal{O},
$$

where $\tau \in \mathbf{R}, g \in L_{l o c}^{2}\left(\mathbf{R}, L^{2}(\mathcal{O})\right)$ and $\mathcal{L}_{K}$ is the non-local operator with the kernel $K$ satisfying (1.5)-(1.7). The nonlinear function $F$ is continuous on $\mathbf{R} \times \mathcal{O} \times \mathbf{R}$ satisfying the following conditions: For all $t, u \in \mathbf{R}$ and $x \in \mathcal{O}$,

$$
\begin{aligned}
& F(t, x, u) u \leqslant-\alpha|u|^{p}+\psi_{1}(t, x), \\
& |F(t, x, u)| \leqslant \psi_{2}(t, x)|u|^{p-1}+\psi_{3}(t, x), \\
& \frac{\partial F}{\partial u}(t, x, u) \leqslant \psi_{4}(t, x),
\end{aligned}
$$

where $p \geqslant 2, \psi_{2}, \psi_{4} \in L^{\infty}(\mathcal{O} \times \mathbf{R}), \psi_{1} \in L_{\mathrm{loc}}^{1}\left(\mathbf{R}, L^{1}(\mathcal{O})\right) \cap L_{\mathrm{loc}}^{\frac{p}{2}}\left(\mathbf{R}, L^{\frac{p}{2}}(\mathcal{O})\right), \psi_{3} \in$ $L_{\text {loc }}^{2}\left(\mathbf{R}, L^{2}(\mathcal{O})\right)$. The noise coefficient is a deterministic function on $\mathcal{O}$ with

$$
\phi \in X_{0}^{s} \cap L^{\infty}(\mathcal{O}), \quad \mathcal{L}_{K} \phi \in L^{2}(\mathcal{O}) .
$$

Then by the interpolation inequality, we immediately have $\phi \in L^{r}(\mathcal{O})$ for any $2 \leqslant r \leqslant$ $\infty$. For the non-autonomous terms $g, \psi_{1}$ and $\psi_{3}$, we impose the following integrability condition: For every $\tau \in \mathbf{R}$,

$$
\int_{-\infty}^{\tau} e^{\lambda_{1} r}\left(\|g(r, .)\|^{2}+\left\|\psi_{1}(r, .)\right\|_{1}+\left\|\psi_{1}(r, .)\right\|_{\frac{p}{2}}^{\frac{p}{2}}+\left\|\psi_{3}(r, .)\right\|^{2}\right) d r<+\infty,
$$

where $\lambda_{1}$ is the first eigenvalue of the non-local operator $\mathcal{L}_{K}$.

Thanks to the Brownian motion $W(t)$ is not differentiable for all most every $t \in \mathbf{R}$, the stochastic equation needs to be transformed into an equation with random coefficient, usually by an Ornstein-Uhlenbeck process over $\left(\Omega, P, \mathcal{F},\left\{\vartheta_{t}\right\}_{t \in \mathbf{R}}\right)$, which is a stationary process

$$
y(t, \omega)=y\left(\vartheta_{t} \omega\right)=-\int_{-\infty}^{0} e^{\xi} \vartheta_{t} \omega(\xi) d \xi, \quad t \in \mathbf{R},
$$


satisfying the stochastic differential equation:

$$
d y\left(\vartheta_{t} \omega\right)+y\left(\vartheta_{t} \omega\right) d t=d W(t) .
$$

Moreover, for any $s, t \in \mathbf{R}$,

$$
y\left(t, \vartheta_{s} \omega\right)=y(t+s, \omega), \quad \text { P-a.s. }
$$

Note that there exists the exceptional set which may be a priori depending on $t, s$. Indeed, we suppose that $y$ has a continuous modification. Once this modification is chosen, the exceptional set is independent of $t$. On the other hand, it is known from $[1,5,4,11]$ that there exits a $\vartheta_{t}$-invariant set $\tilde{\Omega} \subseteq \Omega$ of full measure such that the random variable $|y(\omega)|$ is tempered and $y\left(\vartheta_{t} \omega\right)$ is continuous in $t$ for every fixed $\omega \in \tilde{\Omega}$, and in addition, by Proposition 4.3 .3 in [1], there exists a tempered variable $\varrho(\omega)>0$ such that

$$
|y(\omega)|^{2}+|y(\omega)|^{p}+|y(\omega)|^{2 p-2} \leqslant \varrho(\omega),
$$

where $\varrho(\omega)$ satisfies, for all $\omega \in \tilde{\Omega}$,

$$
\varrho\left(\vartheta_{t} \omega\right) \leqslant \varrho(\omega) e^{\frac{\lambda_{1}}{2}|t|}, \quad t \in \mathbf{R},
$$

where $\lambda_{1}$ is the first eigenvalue of the non-local operator $\mathcal{L}_{K}$. Then it follows from (3.10)-(3.11) that, for all $\omega \in \tilde{\Omega}$,

$$
\left|y\left(\vartheta_{t} \omega\right)\right|^{2}+\left|y\left(\vartheta_{t} \omega\right)\right|^{p}+\left|y\left(\vartheta_{t} \omega\right)\right|^{2 p-2} \leqslant \varrho(\omega) e^{\frac{\lambda_{1}}{2}|t|}, \quad t \in \mathbf{R} .
$$

In what follows, all arguments are understood to hold on this $\tilde{\Omega}$, but for the sake of simplicity we write $\tilde{\Omega}$ as $\Omega$.

Set $z\left(\vartheta_{t} \omega\right)=\phi(x) y\left(\vartheta_{t} \omega\right)$ and write $v(t)=u(t)-z\left(\vartheta_{t} \omega\right)$, where $u(t)$ is the solution to the equations (3.1)-(3.3). Note that the non-local operator $\mathcal{L}_{K}$ is linear, namely, $\mathcal{L}_{K} v(x)=\mathcal{L}_{K} u(x)-\mathcal{L}_{K} z\left(\vartheta_{t} \omega\right)$. Then the stochastic equations (3.1)-(3.3) are transformed into a deterministic one with a parameter $\omega$, namely, $v(t)$ satisfies

$$
\frac{d v}{d t}+\mathcal{L}_{K} v(x)=F\left(t, x, v+z\left(\vartheta_{t} \omega\right)\right)+g(t, x)+z\left(\vartheta_{t} \omega\right)-\mathcal{L}_{K} z\left(\vartheta_{t} \omega\right),
$$

with the homogeneous boundary condition

$$
v(t, x)=0, \quad t>\tau, x \in \mathbf{R}^{N} \backslash \mathcal{O},
$$

and the initial condition

$$
v(\tau, x)=v_{\tau}=u_{\tau}-z\left(\vartheta_{\tau} \omega\right), \quad x \in \mathcal{O} .
$$

By a Galerkin method, similar to [29], one can prove that if $F$ satisfies (3.4)(3.6), then in the case of a bounded domain with Dirichlet boundary condition, for every $\omega \in \Omega$ and $\tau \in \mathbf{R}$, and for $v_{\tau} \in L^{2}$, the problem (3.13)-(3.15) possesses a unique solution $v\left(., \tau, \omega, v_{\tau}\right) \in C\left([\tau, \infty), L^{2}(\mathcal{O})\right) \cap L^{2}\left((\tau, T), X_{0}^{s}\right) \cap L^{p}\left((\tau, T), L^{p}(\mathcal{O})\right)$ with $v\left(\tau, \tau, \omega, v_{\tau}\right)=v_{\tau}$ for all $T>\tau$. Furthermore, this solution operator $v$ is continuous from $L^{2}(\mathcal{O})$ to $L^{2}(\mathcal{O})$ in $v_{\tau}$ and measurable in $\omega$. Let $u\left(t, \tau, \omega, u_{\tau}\right)=$ $v\left(t, \tau, \omega, v_{\tau}\right)+z\left(\vartheta_{t} \omega\right)$. Then formally the process $u$ is the continuous and measurable solution of the problem (3.1)-(3.3) in $L^{2}(\mathcal{O})$.

Given $t \in \mathbf{R}^{+}, \tau \in \mathbf{R}, \omega \in \Omega$ and $u_{\tau} \in L^{2}(\mathcal{O})$, define

$$
\varphi\left(t, \tau, \omega, u_{\tau}\right)=u\left(t+\tau, \tau, \vartheta_{-\tau} \omega, u_{\tau}\right)=v\left(t+\tau, \tau, \vartheta_{-\tau} \omega, v_{\tau}\right)+z\left(\vartheta_{t} \omega\right),
$$

where $u_{\tau}=v_{\tau}+z(\omega)$. Then the random cocycle $\varphi\left(t, \tau, ., u_{\tau}\right)$ is $\left(\mathcal{F}, \mathcal{B}\left(L^{2}(\mathcal{O})\right)\right)$ measurable, and in addition the mapping $\varphi(t, \tau, \omega,):. L^{2}(\mathcal{O}) \rightarrow L^{2}(\mathcal{O})$ is continuous with respect to the initial datum for every $t \in \mathbf{R}^{+}, \tau \in \mathbf{R}$ and $\omega \in \Omega$. Hence, $\varphi$ is 
a continuous random cocycle over the metric dynamical system $\left(\Omega, \mathcal{F}, P,\left\{\vartheta_{t}\right\}_{t \in \mathbf{R}}\right)$ in $L^{2}(\mathcal{O})$.

From now on, we always suppose $\mathfrak{D}$ is a collection of all families of non-empty subsets $D=\left\{D(\tau, \omega) \subset L^{2}(\mathcal{O}): \tau \in \mathbf{R}, \omega \in \Omega\right\}$ such that for each $\tau \in \mathbf{R}$ and $\omega \in \Omega$,

$$
\lim _{t \rightarrow \infty} e^{-\lambda_{1} t}\left\|D\left(\tau-t, \vartheta_{-t} \omega\right)\right\|^{2}=0
$$

where $\|D\|=\sup \{\|u\|: u \in D\}$ and $\lambda_{1}$ is the first eigenvalue of the non-local operator $\mathcal{L}_{K}$. Then it is obvious that $\mathfrak{D}$ is inclusion closed.

Throughout this paper, we denote by $(.,$.$) the inner product in L^{2},\|\cdot\|_{r}$ the norm of the space $L^{r}, 1 \leqslant r \leqslant \infty$, and $r=2$ by $\|$.$\| . In the subsequential discussions,$ we use $c$ to denote the generic positive constant which may depend on the constants $\alpha, s, p, N, \theta$ and $\left\|\psi_{i}\right\|_{\infty}, i=2,4$.

\section{Existence of pullback attractor in $L^{2}(\mathcal{O})$}

In this section, we will derive some uniform estimates of the solutions of the problem (3.1)-(3.3) when the initial datum belongs to $D \in \mathfrak{D}$ and the time $t$ is large enough, which are needed to prove the existence of a $\mathfrak{D}$-pullback random absorbing set and the pullback asymptotical compactness of $\varphi$ defined by $(3.16)$ in $L^{2}(\mathcal{O})$.

Lemma 4.1. Suppose that $F$ satisfies (3.4)-(3.5) and $\mathfrak{D}$ is defined by (3.17), and in addition $\phi \in L^{p}(\mathcal{O}) \cap X_{0}^{s}$ and (3.8) holds. Let $\tau \in \mathbf{R}, \omega \in \Omega$ and $D=$ $\{D(\tau, \omega): \tau \in \mathbf{R}, \omega \in \Omega\} \in \mathfrak{D}$ with $u_{\tau-t} \in D\left(\tau-t, \vartheta_{-t} \omega\right)$. Then there exists a constant $T=T(\tau, \omega, D) \geqslant 1$ such that for all $t \geqslant T$, the solution $u$ of problem (3.1)-(3.3) satisfies

$$
\left\|u\left(\sigma, \tau-t, \vartheta_{-\tau} \omega, u_{\tau-t}\right)\right\|^{2} \leqslant c(1+C(\tau, \omega)), \quad \sigma \in[\tau-1, \tau],
$$

and

$$
\int_{\tau-t}^{\tau} e^{\lambda_{1}(r-\tau)}\left(\|v(r)\|_{X_{0}^{s}}^{2}+\|v(r)\|_{p}^{p}\right) d r \leqslant c(1+C(\tau, \omega)),
$$

where $c>0$ is a deterministic constant and

$$
C(\tau, \omega) \triangleq \varrho(\omega)+\int_{-\infty}^{0} e^{\lambda_{1} r}\left(\|g(r+\tau, .)\|^{2}+\left\|\psi_{1}(r+\tau, .)\right\|_{1}+\left\|\psi_{3}(r+\tau, .)\right\|^{2}\right) d r .
$$

Proof. Using the test function $v$ in (3.13), we obtain

$$
\begin{aligned}
\frac{1}{2} & \frac{d}{d t}\|v\|^{2}+\int_{\mathbf{R}^{2 N}}|v(x)-v(y)|^{2} K(x-y) d x d y \\
= & \int_{\mathcal{O}} F\left(t, x, v+z\left(\vartheta_{t} \omega\right)\right) v d x+\int_{\mathcal{O}} g(t, x) v d x+\int_{\mathcal{O}} z\left(\vartheta_{t} \omega\right) v d x \\
& -\int_{\mathbf{R}^{2 N}} y\left(\vartheta_{t} \omega\right)(\phi(x)-\phi(y))(v(x)-v(y)) K(x-y) d x d y .
\end{aligned}
$$

We now estimate every term on the right hand side of (4.3). First for the nonlinearity, in view of (3.4) and (3.5), by using Young inequality repeatedly, we have

$$
\begin{aligned}
F\left(t, x, v+z\left(\vartheta_{t} \omega\right)\right) v & =F(t, x, u) u-F(t, x, u) z\left(\vartheta_{t} \omega\right) \\
& \leqslant-\alpha|u|^{p}+\psi_{1}+\psi_{2}|u|^{p-1}\left|z\left(\vartheta_{t} \omega\right)\right|+\psi_{3}\left|z\left(\vartheta_{t} \omega\right)\right|
\end{aligned}
$$




$$
\begin{aligned}
& \leqslant-\frac{1}{2} \alpha|u|^{p}+\psi_{1}+2^{p-1} \psi_{2}^{p}\left|z\left(\vartheta_{t} \omega\right)\right|^{p}+\psi_{3}\left|z\left(\vartheta_{t} \omega\right)\right| \\
& \leqslant-\frac{1}{2^{p}} \alpha|v|^{p}+\left(\frac{1}{2} \alpha+2^{p-1} \psi_{2}^{p}\right)\left|z\left(\vartheta_{t} \omega\right)\right|^{p}+\left|z\left(\vartheta_{t} \omega\right)\right|^{2}+\psi_{1}+\left|\psi_{3}\right|^{2},
\end{aligned}
$$

where we used the inequality $|a+b|^{r} \geqslant 2^{1-r}|a|^{r}-|b|^{r}$ for any $r \geqslant 1$ in the first term of the last inequality on the right hand side of (4.4). By (3.7), we deduce from (4.4) that

$$
\begin{aligned}
& \int_{\mathcal{O}} F(t, x, u) v d x \\
& \leqslant-\frac{\alpha}{2^{p}}\|v\|_{p}^{p}+c\left(\left|y\left(\vartheta_{t} \omega\right)\right|^{2}+\left|y\left(\vartheta_{t} \omega\right)\right|^{p}\right)+\left\|\psi_{1}(t, .)\right\|_{1}+\left\|\psi_{3}(t, .)\right\|^{2} .
\end{aligned}
$$

On the other hand, by Young inequality, along with $\phi \in X_{0}^{s}$, we have

$$
\begin{aligned}
& \left|\int_{\mathbf{R}^{2 N}} y\left(\vartheta_{t} \omega\right)(\phi(x)-\phi(y))(v(x)-v(y)) K(x-y) d x d y\right| \\
& \leqslant \frac{1}{8} \int_{\mathbf{R}^{2 N}}|v(t)(x)-v(t)(y)|^{2} K(x-y) d x d y \\
& \quad+2\left|y\left(\vartheta_{t} \omega\right)\right|^{2} \int_{\mathbf{R}^{2 N}}|\phi(x)-\phi(y)|^{2} K(x-y) d x d y \\
& \leqslant \frac{1}{8} \int_{\mathbf{R}^{2 N}}|v(t)(x)-v(t)(y)|^{2} K(x-y) d x d y+c\left|y\left(\vartheta_{t} \omega\right)\right|^{2},
\end{aligned}
$$

where $c=c\left(\|\phi\|_{X_{0}^{s}}\right)>0$. In addition, by $g \in L_{l o c}^{2}\left(\mathbf{R}, L^{2}(\mathcal{O})\right)$, we infer that

$$
\left|\int_{\mathcal{O}} g(t, x) v d x+\int_{\mathcal{O}} z\left(\vartheta_{t} \omega\right) v d x\right| \leqslant \frac{\lambda_{1}}{4}\|v\|^{2}+c\|g(t, .)\|^{2}+c\left|y\left(\vartheta_{t} \omega\right)\right|^{2}
$$

where $\lambda_{1}$ is the first eigenvalue of the non-local operator $\mathcal{L}_{K}$ and $c=c\left(\lambda_{1},\|\phi\|\right)$. By a combination of (4.5) and (4.6)-(4.7) into (4.3), it follows that

$$
\begin{aligned}
& \frac{1}{2} \frac{d}{d t}\|v\|^{2}+\frac{7}{8} \int_{\mathbf{R}^{2 N}}|v(t)(x)-v(t)(y)|^{2} K(x-y) d x d y+\frac{\alpha}{2^{p}}\|v\|_{p}^{p} \\
& \leqslant \frac{\lambda_{1}}{4}\|v\|^{2}+J\left(\vartheta_{t} \omega\right)+\left\|\psi_{1}(t, .)\right\|_{1}+\left\|\psi_{3}(t, .)\right\|^{2}+c\|g(t, .)\|^{2} .
\end{aligned}
$$

Using Lemma 2.2 (i), we have

$$
\begin{aligned}
& \frac{7}{8} \int_{\mathbf{R}^{2 N}}|v(t)(x)-v(t)(y)|^{2} K(x-y) d x d y \\
& \geqslant \frac{1}{8} \int_{\mathbf{R}^{2 N}}|v(t)(x)-v(t)(y)|^{2} K(x-y) d x d y+\frac{6}{8} \lambda_{1}\|v\|^{2} \\
& =\frac{1}{8}\|v\|_{X_{0}^{s}}+\frac{3}{4} \lambda_{1}\|v\|^{2} .
\end{aligned}
$$

Consequently, by (4.8) and (4.9), we derive the energy inequality:

$$
\begin{aligned}
& \frac{d}{d t}\|v\|^{2}+\lambda_{1}\|v\|^{2}+\frac{1}{4}\|v\|_{X_{0}^{s}}^{2}+\frac{\alpha}{2^{p-1}}\|v\|_{p}^{p} \\
& \leqslant J\left(\vartheta_{t} \omega\right)+2\left\|\psi_{1}(t, .)\right\|_{1}+2\left\|\psi_{3}(t, .)\right\|^{2}+2 c\|g(t, .)\|^{2}
\end{aligned}
$$


where $J\left(\vartheta_{t} \omega\right) \triangleq c\left(\left|y\left(\vartheta_{t} \omega\right)\right|^{2}+\left|y\left(\vartheta_{t} \omega\right)\right|^{p}\right)$ with $c=c\left(\alpha, p,\|\phi\|,\|\phi\|_{p},\|\phi\|_{X_{0}^{s}}\right)$. Using the classic Gronwall lemma to (4.10) over the interval $[\tau-t, \sigma]$ with $\sigma \in[\tau-1, \tau]$ and $t \geqslant 1$, meanwhile, using $\vartheta_{-\tau} \omega$ to replace $\omega$, we deduce that

$$
\begin{aligned}
& \|v(\sigma)\|^{2}+\int_{\tau-t}^{\sigma} e^{\lambda_{1}(r-\sigma)}\left(\frac{1}{4}\|v(r)\|_{X_{0}^{s}}^{2}+\frac{\alpha}{2^{p-1}}\|v(r)\|_{p}^{p}\right) d r \\
& \leqslant e^{\lambda_{1}}\left(e^{-\lambda_{1} t}\left\|v_{\tau-t}\right\|^{2}+\int_{\tau-t}^{\tau} e^{\lambda_{1}(r-\tau)} J\left(\vartheta_{r-\tau} \omega\right) d r\right. \\
& \left.\quad+c \int_{\tau-t}^{\tau} e^{\lambda_{1}(r-\tau)}\left(\|g(r, .)\|^{2}+\left\|\psi_{1}(r, .)\right\|_{1}+\left\|\psi_{3}(r, .)\right\|^{2}\right) d r\right),
\end{aligned}
$$

where $v($.$) is the abbreviation of v\left(., \tau-t, \vartheta_{-\tau} \omega, v_{\tau-t}\right)$ and $c=c\left(\lambda_{1}\right)$. By (3.12) we have $J\left(\vartheta_{r-\tau} \omega\right) \leqslant e^{\frac{\lambda_{1}}{2}|r-\tau|} \varrho(\omega)$, and thereby we may rewrite (4.11) as the following

$$
\begin{aligned}
& \|v(\sigma)\|^{2}+\int_{\tau-t}^{\sigma} e^{\lambda_{1}(r-\sigma)}\left(\frac{1}{4}\|v(r)\|_{X_{0}^{s}}^{2}+\frac{\alpha}{2^{p-1}}\|v(r)\|_{p}^{p}\right) d r \\
& \leqslant e^{\lambda_{1}}\left(e^{-\lambda_{1} t}\left\|v_{\tau-t}\right\|^{2}+\varrho(\omega) \int_{\tau-t}^{\tau} e^{\frac{1}{2} \lambda_{1}(r-\tau)} d r\right. \\
& \left.\quad+c \int_{\tau-t}^{\tau} e^{\lambda_{1}(r-\tau)}\left(\|g(r, .)\|^{2}+\left\|\psi_{1}(r, .)\right\|_{1}+\left\|\psi_{3}(r, .)\right\|^{2}\right) d r\right) \\
& \leqslant e^{\lambda_{1}}\left(2 e^{-\lambda_{1} t}\left(\left\|u_{\tau-t}\right\|^{2}+\|\phi\|^{2}\left|z\left(\vartheta_{-t} \omega\right)\right|^{2}\right)+\frac{2}{\lambda_{1}} \varrho(\omega)\right. \\
& \left.\quad+c \int_{-\infty}^{0} e^{\lambda_{1} r}\left(\|g(r+\tau, .)\|^{2}+\left\|\psi_{1}(r+\tau, .)\right\|_{1}+\left\|\psi_{3}(r+\tau, .)\right\|^{2}\right) d r\right) .
\end{aligned}
$$

By noting that $u_{\tau-t} \in D\left(\tau-t, \vartheta_{-t} \omega\right)$ and $|y(\omega)|$ is tempered, we have

$$
2 e^{-\lambda_{1} t}\left(\left\|u_{\tau-t}\right\|^{2}+\|\phi\|^{2}\left|y\left(\vartheta_{-t} \omega\right)\right|^{2}\right) \rightarrow 0,
$$

as $t \rightarrow \infty$. By (3.8), deduce that $c \int_{-\infty}^{0} e^{\lambda_{1} r}\left(\|g(r+\tau, .)\|^{2}+\left\|\psi_{1}(r+\tau, .)\right\|_{1}+\| \psi_{3}(r+\right.$ $\left.\tau,.) \|^{2}\right) d r<\infty$. Therefore, it follows from (4.12) and (4.13) that, for every fixed $\tau \in \mathbf{R}, \omega \in \Omega$ and all $u_{\tau-t} \in D\left(\tau-t, \vartheta_{-t} \omega\right)$, there exists a $T=T(\tau, \omega, D) \geqslant 1$ such that for all $t \geqslant T$,

$$
\begin{aligned}
& \|v(\sigma)\|^{2}+\int_{\tau-t}^{\sigma} e^{\lambda_{1}(r-\sigma)}\left(\frac{1}{4}\|v\|_{X_{0}^{s}}^{2}+\frac{\alpha}{2^{p-1}}\|v(r)\|_{p}^{p}\right) d r \\
& \leqslant c\left(1+\varrho(\omega)+\int_{-\infty}^{0} e^{\lambda_{1} r}\left(\|g(r+\tau, .)\|^{2}+\left\|\psi_{1}(r+\tau, .)\right\|_{1}\right.\right. \\
& \left.\left.\quad+\left\|\psi_{3}(r+\tau, .)\right\|^{2}\right) d r\right) \triangleq c(1+C(\tau, \omega)),
\end{aligned}
$$

where

$$
C(\tau, \omega) \triangleq \varrho(\omega)+\int_{-\infty}^{0} e^{\lambda_{1} r}\left(\|g(r+\tau, .)\|^{2}+\left\|\psi_{1}(r+\tau, .)\right\|_{1}+\left\|\psi_{3}(r+\tau, .)\right\|^{2}\right) d r<\infty .
$$

On the other hand, in view of $u(t)=v(t)+z\left(\vartheta_{t} \omega\right)$ and by (3.12) it follows that, for $\sigma \in[\tau-1, \tau]$

$$
\begin{aligned}
\|u(\sigma)\|^{2} & \leqslant 2\|v(\sigma)\|^{2}+2\|\phi\|^{2}\left|y\left(\vartheta_{\sigma-\tau} \omega\right)\right|^{2} \leqslant 2\|v(\sigma)\|^{2}+2\|\phi\|^{2} \varrho(\omega) e^{\frac{\lambda_{1}}{2}|\sigma-\tau|} \\
& \leqslant 2\|v(\sigma)\|^{2}+2\|\phi\|^{2} \varrho(\omega) e^{\frac{\lambda_{1}}{2}} .
\end{aligned}
$$

Then the needed estimates are derived from (4.14) and (4.15). 
In order to prove the asymptotical compactness, we first prove a convergence result of the solutions to the problem (3.1)-(3.3) if the initial data sequence is uniformly bounded in $L^{2}(\mathcal{O})$.

Lemma 4.2. Suppose that $\left\{x_{n}\right\}_{n \in \mathbf{N}} \subset L^{2}(\mathcal{O})$ is an initial values sequence with $\sup _{n}\left\|x_{n}\right\| \leqslant M$ for some fixed $M>0$. Let $\tau \in \mathbf{R}$ and $\omega \in \Omega$. Then for almost every $\sigma \in[\tau-1, \tau]$, there exits a fixed point $u_{\sigma} \in L^{2}(\mathcal{O})$ such that the sequence $\left\{u_{n}\left(\sigma, \tau-1, \omega, x_{n}\right)\right\}_{n \in \mathbf{N}}$ has a convergent subsequence with $u_{n}\left(\sigma, \tau-1, \omega, x_{n}\right) \rightarrow u_{\sigma}$ in $L^{2}(\mathcal{O})$, where $u_{n}\left(\sigma, \tau-1, \omega, x_{n}\right)$ is a solution to (3.1)-(3.3) corresponding the initial value $x_{n}$.

Proof. Given $n \in \mathbf{N}$, let $v_{n}(t)$ be a solution to (3.13)-(3.15) corresponding the initial datum $x_{n}-\phi z\left(\vartheta_{\tau-1} \omega\right)$ at the initial time $\tau-1$. From (4.10), we have for any $t \geqslant \tau-1$,

$$
\frac{d}{d t}\left\|v_{n}(t)\right\|^{2}+\frac{1}{4}\left\|v_{n}(t)\right\|_{X_{0}^{s}}^{2} \leqslant 2 J\left(\vartheta_{t} \omega\right)+2\left\|\psi_{1}(t, .)\right\|_{1}+2\left\|\psi_{3}(t, .)\right\|^{2}+c\|g(t, .)\|^{2} .
$$

Integrate (4.16) from $\tau-1$ to $\sigma$ for $\sigma \in[\tau-1, \tau]$ to find that

$$
\begin{aligned}
\| v_{n}\left(\sigma, \tau-1, \omega, x_{n}-\right. & \left.\phi z\left(\vartheta_{\tau-1} \omega\right)\right)\left\|^{2}+\frac{1}{4} \int_{\tau-1}^{\tau}\right\| v_{n}\left(r, \tau-1, \omega, x_{n}-\phi z\left(\vartheta_{\tau-1} \omega\right)\right) \|_{X_{0}^{s}}^{2} d r \\
\leqslant & 2\left(\left\|x_{n}\right\|^{2}+\|\phi\|^{2}\left|z\left(\vartheta_{\tau-1} \omega\right)\right|^{2}\right)+c \int_{\tau-1}^{\tau}\left(J\left(\vartheta_{r} \omega\right)\right. \\
& \left.+\|g(r, .)\|^{2}+\left\|\psi_{1}(r, .)\right\|_{1}+\left\|\psi_{3}(r, .)\right\|^{2}\right) d r .
\end{aligned}
$$

Consider that $g \in L_{l o c}^{2}\left(\mathbf{R}, L^{2}(\mathcal{O})\right), \phi \in X_{0}^{s}, J\left(\vartheta_{r} \omega\right)$ is continuous in $r \in \mathbf{R}$ and $u_{n}(t)=v_{n}(t)+z\left(\vartheta_{t} \omega\right)$. Then we deduce from (4.17) that for every $\sigma \in[\tau-1, \tau]$,

$$
\left\|u_{n}\left(\sigma, \tau-1, \omega, x_{n}\right)\right\|^{2} \leqslant C_{0}(\tau, \omega)
$$

and

$$
\int_{\tau-1}^{\tau}\left\|u_{n}\left(r, \tau-1, \omega, x_{n}\right)\right\|_{X_{0}^{s}}^{2} d r \leqslant C_{0}(\tau, \omega),
$$

where $C_{0}(\tau, \omega)<\infty$ is a positive random constant independent of $n$. According to (4.18), we have that for every $\sigma \in[\tau-1, \tau]$, there exist $u_{\sigma} \in L^{2}(\mathcal{O})$ and a subsequence (which is not relabeled) by a diagonal process such that

$$
u_{n}\left(\sigma, \tau-1, \omega, x_{n}\right) \rightarrow u_{\sigma} \text { weakly in } L^{2}(\mathcal{O}) .
$$

In view of (4.19) we infer that

(4.21) $u_{n}\left(., \tau-1, \omega, x_{n}\right)$ is unformly bounded with respect to $n$ in $L^{2}\left(\tau-1, \tau ; X_{0}^{s}\right)$.

But by Lemma $2.1(i v)$, the embedding of $X_{0}^{s} \hookrightarrow L^{2}(\mathcal{O})$ is compact and therefore from (4.21), there is a subsequence (not labeled again) by a diagonal process such that

$$
\left\{u_{n}\left(., \tau-1, \omega, x_{n}\right)\right\}_{n=1}^{\infty} \text { strongly converges in } L^{2}\left(\tau-1, \tau ; L^{2}(\mathcal{O})\right) .
$$

This along with (4.20) implies that for all most every $\sigma \in[\tau-1, \tau]$,

$$
u_{n}\left(\sigma, \tau-1, \omega, x_{n}\right) \rightarrow u_{\sigma} \text { strongly in } L^{2}(\mathcal{O}),
$$

which proves our claim.

Based on the above lemma and in conjunction with the cocycle property of $\varphi$ and the continuity of solutions, we can prove the asymptotical compactness of random cocycle $\varphi$ in $L^{2}(\mathcal{O})$. 
Lemma 4.3. Suppose that the nonlinearity $F$ satisfies (3.4)-(3.6) and $\mathfrak{D}$ is defined by (3.17), in addition $\phi \in L^{p}(\mathcal{O}) \cap X_{0}^{s}$ and (3.8) holds. Then the random cocycle $\varphi$ defined by (3.16) is $\mathfrak{D}$-pullback asymptotically compact in $L^{2}(\mathcal{O})$, namely, for every $\tau \in \mathbf{R}$ and $\omega \in \Omega$, the sequence $\left\{\varphi\left(t_{n}, \tau-t_{n}, \vartheta_{-t_{n}} \omega, u_{0, n}\right)\right\}_{n=1}^{\infty}$ is precompact in $L^{2}(\mathcal{O})$ whenever $t_{n} \rightarrow \infty$ and $u_{0, n} \in D\left(\tau-t_{n}, \vartheta_{-t_{n}} \omega\right)$ with $D \in \mathfrak{D}$.

Proof. Given $\tau \in \mathbf{R}, \omega \in \Omega$ and $u_{0, n} \in D\left(\tau-t_{n}, \vartheta_{-t_{n}} \omega\right)$. By Lemma 4.1, we have

$$
\left\|u\left(\tau-1, \tau-t_{n}, \vartheta_{-\tau} \omega, u_{0, n}\right)\right\|^{2} \leqslant c(1+C(\tau, \omega)),
$$

when $n$ is large enough. We now put $x_{n}=u\left(\tau-1, \tau-t_{n}, \vartheta_{-\tau} \omega, u_{0, n}\right)$ and then utilize Lemma 4.2 to find that there is a certain real number $\sigma \in[\tau-1, \tau], u_{\sigma} \in L^{2}(\mathcal{O})$ and up to a subsequence (not relabeled again) such that

$$
u\left(\sigma, \tau-1, \vartheta_{-\tau} \omega, u\left(\tau-1, \tau-t_{n}, \vartheta_{-\tau} \omega, u_{0, n}\right)\right) \rightarrow u_{\sigma} \text { strongly in } L^{2}(\mathcal{O}) .
$$

Then (4.24) implies that

$$
u\left(\sigma, \tau-t_{n}, \vartheta_{-\tau} \omega, u_{0, n}\right) \rightarrow u_{\sigma} \text { strongly in } L^{2}(\mathcal{O}) .
$$

Noting that

$$
u\left(\tau, \tau-t_{n}, \vartheta_{-\tau} \omega, u_{0, n}\right)=u\left(\tau, \sigma, \vartheta_{-\tau} \omega, u\left(\sigma, \tau-t_{n}, \vartheta_{-\tau} \omega, u_{0, n}\right)\right),
$$

and in conjunction with the continuity of solution in $L^{2}(\mathcal{O})$ with respect to the initial data, we deduce that, for this fixed $\sigma \in[\tau-1, \tau]$,

$$
u\left(\tau, \tau-t_{n}, \vartheta_{-\tau} \omega, u_{0, n}\right) \rightarrow u\left(\tau, \sigma, \vartheta_{-\tau} \omega, u_{\sigma}\right) \text { strongly in } L^{2}(\mathcal{O}) .
$$

Consider that $\varphi\left(t_{n}, \tau-t_{n}, \vartheta_{-t_{n}} \omega, u_{0, n}\right)=u\left(\tau, \tau-t_{n}, \vartheta_{-\tau} \omega, u_{0, n}\right)$ and consequently $\varphi$ is asymptotically compact in $L^{2}(\mathcal{O})$.

We next prove the existence of $\mathfrak{D}$-pullback absorbing set for the random cocycle $\varphi$ defined by $(3.16)$ in $L^{2}(\mathcal{O})$.

Lemma 4.4. Suppose that the nonlinearity $F$ satisfies (3.4)-(3.6) and $\mathfrak{D}$ is defined by (3.17), in addition $\phi \in L^{p}(\mathcal{O}) \cap X_{0}^{s}$ and (3.8) holds. Define

$$
\mathcal{K}(\tau, \omega)=\left\{u \in L^{2}(\mathcal{O}):\|u\|^{2} \leqslant c(1+C(\tau, \omega))\right\}, \quad \tau \in \mathbf{R}, \omega \in \Omega,
$$

where $C(\tau, \omega)$ is the same as Lemma 4.1. Then the family $\mathcal{K}=\{\mathcal{K}(\tau, \omega): \tau \in \mathbf{R}, \omega \in$ $\Omega\} \in \mathfrak{D}$ is a closed and measurable $\mathfrak{D}$-pullback absorbing set in $L^{2}(\mathcal{O})$ for the random cocycle $\varphi$ defined by (3.16).

Proof. First, by (3.8) and the tempered property of $\varrho(\omega)$, it is clear that for each $\tau \in \mathbf{R}$ and $\omega \in \Omega, \lim _{t \rightarrow \infty} e^{-\lambda_{1} t} C\left(\tau-t, \vartheta_{-t} \omega\right)=0$. Then by (3.17), the family $\mathcal{K} \in \mathfrak{D}$. On the other hand, for any $x \in L^{2}(\mathcal{O})$ and a fixed $\tau \in \mathbf{R}$, we have

$$
d_{L^{2}(\mathcal{O})}(x, \mathcal{K}(\tau, \omega))=\inf _{z \in \mathcal{K}(\tau, \omega)} d_{L^{2}(\mathcal{O})}(x, z)=\|x\|+\left(c(1+C(\tau, \omega))^{\frac{1}{2}}\right.
$$

is $(\mathcal{F}, \mathcal{B}(\mathbf{R}))$-measurable as a random variable. Then the set-valued mapping $\mathcal{K}$ defined by (4.27) is a closed random absorbing set in $L^{2}(\mathcal{O})$ in the sense of Definition 2.3, which completes the proof.

By Lemma 4.3 and 4.4, we are at the point to present the existence of $\mathfrak{D}$-pullback attractor for the problem $(3.1)-(3.3)$ in $L^{2}(\mathcal{O})$.

Theorem 4.5. Let $s \in(0,1)$ and $N>2 s$. Suppose that the nonlinearity $F$ satisfies (3.4)-(3.6) and $\mathfrak{D}$ is defined by (3.17), in addition $\phi \in L^{p}(\mathcal{O}) \cap X_{0}^{s}$ and (3.8) holds. Then the random cocycle $\varphi$ defined by (3.16) admits a unique $\mathfrak{D}$-pullback 
attractor $\mathcal{A}=\{\mathcal{A}(\tau, \omega): \tau \in \mathbf{R}, \omega \in \Omega\}$ in $L^{2}(\mathcal{O})$, where the component $\mathcal{A}(\tau, \omega)$ is defined as

$$
\mathcal{A}(\tau, \omega) \triangleq \bigcap_{s \geqslant 0} \overline{\bigcup_{t \geqslant s} \varphi\left(t, \tau-t, \vartheta_{-t} \omega, \mathcal{K}\left(\tau-t, \vartheta_{-t} \omega\right)\right)} .
$$

Proof. By Lemma 4.3 and Lemma 4.4, we get immediately from the fist part of Theorem 2.8 that $\mathcal{A}$ is a $\mathfrak{D}$-pullback attractor for $\varphi$ in $L^{2}(\mathcal{O})$.

Remark 2. If in particular, the kernel $K(x)=|x|^{-(N+2 s)}$, then as a direct consequence of Theorem 4.5, we get that the fractional Lapacian model (1.9) admits a unique pullback attractor in $L^{2}(\mathcal{O})$. The reader is referred to [29] for the studying of (1.9) with multiplicative noise.

\section{Regular dynamics in $X_{0}^{s}$}

In order to derive the regular dynamics of solutions to (3.1)-(3.3) in the regular space $X_{0}^{s}$, it suffices to check that the cocycle is asymptotically compact in $X_{0}^{s}$. There are in general two ways to attack the regular problems. One is to use the spectrum splitting (cf. [13]) and truncation estimate method (cf. [31]), and another is to prove the continuity of solutions with respect to the initial datum in the corresponding space (cf. [32, 30, 33]). In this paper, we adapt the spectrum splitting and truncation method to cope with the non-local fractional equation (1.1), by which one of the crucial keys is to check the flattening condition. For this purpose, we need to prove a series of a priori estimates of solutions.

For convenience, we present the following lemma which is repeatedly used in what follows.

Lemma 5.1. [32] Let $y, g$ and $h$ be tree nonnegative and locally integrable functions on $\mathbf{R}$ such that $\frac{d y}{d t}$ is also locally integrable and

$$
\frac{d y(t)}{d t}+\nu y(t)+g(t) \leqslant h(t), \quad t \in \mathbf{R}
$$

for some constant $\nu \in \mathbf{R}^{+}$. Then

(i) for arbitrary $r>0$ and $\tau \in \mathbf{R}$,

$$
y(\tau) \leqslant \frac{1}{r} \int_{\tau-r}^{\tau} e^{\nu(t-\tau)} y(t) d t+\int_{\tau-r}^{\tau} e^{\nu(t-\tau)} h(t) d t
$$

(ii) for arbitrary $r, \epsilon>0$ and $\sigma \in[\tau-r, \tau]$,

$$
\begin{aligned}
& y(\sigma)+\int_{\tau-r}^{\tau} e^{\nu(t-\tau)} g(t) d t \\
& \leqslant \frac{e^{\nu r}+1}{\epsilon} \int_{\tau-r-\epsilon}^{\tau} e^{\nu(t-\tau)} y(t) d t+\left(e^{\nu r}+2\right) \int_{\tau-r-\epsilon}^{\tau} e^{\nu(t-\tau)} h(t) d t .
\end{aligned}
$$

In particular, this holds for $\nu=0$.

5.1. A priori estimates of solutions. We now begin with a priori $L^{2 p-2}$ estimate of solutions on the intervals $[\tau-2, \tau]$.

Lemma 5.2. Suppose that (3.4)-(3.8) and $\mathfrak{D}$ is defined by (3.17). Let $\tau \in$ $\mathbf{R}, \omega \in \Omega$ and $D=\{D(\tau, \omega): \tau \in \mathbf{R}, \omega \in \Omega\} \in \mathfrak{D}$ with $u_{\tau-t} \in D\left(\tau-t, \vartheta_{-t} \omega\right)$. Then there exist constants $M=M(\tau, \omega)>0$ and $T=T(\tau, \omega, D) \geqslant 3$ such that for all 
$t \geqslant T$, the solution $u$ of problem (3.13)-(3.15) satisfies

$$
\left\|v\left(\sigma, \tau-t, \vartheta_{-\tau} \omega, v_{\tau-t}\right)\right\|_{p}^{p} \leqslant M(\tau, \omega), \quad \sigma \in[\tau-2, \tau],
$$

and

$$
\int_{\tau-2}^{\tau}\left\|v\left(\xi, \tau-t, \vartheta_{-\tau} \omega, v_{\tau-t}\right)\right\|_{2 p-2}^{2 p-2} d \xi \leqslant M(\tau, \omega)
$$

where $v_{\tau-t}=u_{\tau-t}-z\left(\vartheta_{-t} \omega\right)$ and $M(\tau, \omega)$ is a constant depending $\alpha, p, \lambda_{1},\|\phi\|$, $\|\phi\|_{2 p-2}, \quad\left\|\mathcal{L}_{K} \phi\right\|, \quad\left\|\psi_{2}\right\|_{\infty}, \quad C(\tau, \omega), \quad \int_{\tau-3}^{\tau}\|g(\xi, .)\|^{2} d \xi, \quad \int_{\tau-3}^{\tau}\left\|\psi_{1}(\xi, .)\right\|_{\frac{p}{2}}^{\frac{p}{2}} d \xi \quad$ and $\int_{\tau-3}^{\tau}\left\|\psi_{3}(\xi, .)\right\|^{2} d \xi$

Proof. By (3.13), using the test function $|v|^{p-2} v$, we have

$$
\begin{aligned}
\frac{1}{p} \frac{d}{d t}\|v\|_{p}^{p}+\left(\mathcal{L}_{K} v,|v|^{p-2} v\right)= & \int_{\mathcal{O}} F(t, x, u)|v|^{p-2} v d x+\int_{\mathcal{O}} g(t, x)|v|^{p-2} v d x \\
& +\int_{\mathcal{O}} z\left(\vartheta_{t} \omega\right)|v|^{p-2} v d x-\left(\mathcal{L}_{K} z\left(\vartheta_{t} \omega\right),|v|^{p-2} v\right),
\end{aligned}
$$

where we can easily obtain

$$
\begin{aligned}
\left(\mathcal{L}_{K} v,|v|^{p-2} v\right) & =\int_{\mathbf{R}^{2 N}}(v(x)-v(y))\left(|v(x)|^{p-2} v(x)-|v(y)|^{p-2} v(y)\right) K(x-y) d x d y \\
& \geqslant c(p) \int_{\mathbf{R}^{2 N}}|v(x)-v(y)|^{p} K(x-y) d x d y \geqslant 0
\end{aligned}
$$

and

$$
\begin{aligned}
& \int_{\mathcal{O}} g(t, x)|v|^{p-2} v d x+\int_{\mathcal{O}} z\left(\vartheta_{t} \omega\right)|v|^{p-2} v d x \\
& \leqslant \frac{\alpha}{2^{p+2}}\|v\|_{2 p-2}^{2 p-2}+c\left(\|g(t, .)\|^{2}+\left|y\left(\vartheta_{t} \omega\right)\right|^{2}\right),
\end{aligned}
$$

where $c=c(p, \alpha,\|\phi\|)$. It remains to estimate the nonlinearity and the last term on the right hand side of (5.3). From (4.4), we infer that

$$
F\left(t, x, v+z\left(\vartheta_{t} \omega\right)\right) v \leqslant-\frac{1}{2^{p}} \alpha|v|^{p}+c\left|z\left(\vartheta_{t} \omega\right)\right|^{p}+\psi_{1}+\psi_{3}\left|z\left(\vartheta_{t} \omega\right)\right|,
$$

where $c=c\left(\alpha, p,\left\|\psi_{2}\right\|_{\infty}\right)$. By using Young inequality repeatedly, we deduce from (5.6) that

$$
\begin{aligned}
\int_{\mathcal{O}} F(t, x, u)|v|^{p-2} v d x \leqslant & -\frac{\alpha}{2^{p+1}}\|v\|_{2 p-2}^{2 p-2}+c\|v\|_{p}^{p}+c\left|y\left(\vartheta_{t} \omega\right)\right|^{2 p-2} \\
& +c\left(\left\|\psi_{1}(t, .)\right\|_{\frac{p}{2}}^{\frac{p}{2}}+\left\|\psi_{3}(t, .)\right\|^{2}\right)
\end{aligned}
$$

where $c=c\left(\alpha, p,\left\|\psi_{2}\right\|_{\infty},\|\phi\|_{2 p-2}\right)$. Indeed, to derive the estimate of the last term in (5.7), we used

$$
\begin{aligned}
\int_{\mathcal{O}} \psi_{3}\left|z\left(\vartheta_{t} \omega\right) \| v\right|^{p-2} d x & \leqslant\left\|\psi_{3}(t, .)\right\|^{2}+\int_{\mathcal{O}}\left|z\left(\vartheta_{t} \omega\right)\right|^{2}|v|^{2 p-4} d x \\
& \leqslant\left\|\psi_{3}(t, .)\right\|^{2}+\frac{\alpha}{2^{p+3}}\|v\|_{2 p-2}^{2 p-2}+c\left\|z\left(\vartheta_{t} \omega\right)\right\|_{2 p-2}^{2 p-2} .
\end{aligned}
$$


On the other hand, by using Young inequality again we get that

$$
\begin{aligned}
& \left(\mathcal{L}_{K} z\left(\vartheta_{t} \omega\right),|v|^{p-2} v\right) \\
& =\int_{\mathbf{R}^{2 N}} y\left(\vartheta_{t}\right)(\phi(x)-\phi(y))\left(|v(x)|^{p-2} v(x)-|v(y)|^{p-2} v(y)\right) K(x-y) d x d y \\
& =\int_{\mathbf{R}^{2 N}} y\left(\vartheta_{t} \omega\right)(\phi(x)-\phi(y))|v(x)|^{p-2} v(x) K(x-y) d x d y \\
& \quad-\int_{\mathbf{R}^{2 N}} y\left(\vartheta_{t} \omega\right)(\phi(x)-\phi(y))|v(y)|^{p-2} v(y) K(x-y) d x d y .
\end{aligned}
$$

Thanks to $\mathcal{L}_{K} \phi \in L^{2}$, by Hölder inequality, we have

$$
\begin{aligned}
& \int_{\mathbf{R}^{2 N}} y\left(\vartheta_{t} \omega\right)(\phi(x)-\phi(y))|v(x)|^{p-2} v(x) K(x-y) d x d y \\
& \leqslant\left\{\int_{\mathbf{R}^{N}}\left(\int_{\mathbf{R}^{N}} y\left(\vartheta_{t} \omega\right)(\phi(x)-\phi(y)) K(x-y) d y\right)^{2} d x\right\}^{\frac{1}{2}}\left(\int_{\mathcal{O}}|v(x)|^{2 p-2} d x\right)^{\frac{1}{2}} \\
& \leqslant \frac{\alpha}{2^{p+3}}\|v\|_{2 p-2}^{2 p-2}+c\left|y\left(\vartheta_{t} \omega\right)\right|^{2} .
\end{aligned}
$$

Hence, it follows from (5.8) and 5.9) that

$$
-\left(\mathcal{L}_{K} z\left(\vartheta_{t} \omega\right),|v|^{p-2} v\right) \leqslant \frac{\alpha}{2^{p+2}}\|v\|_{2 p-2}^{2 p-2}+c\left|y\left(\vartheta_{t} \omega\right)\right|^{2} .
$$

Then combine (5.3)-(5.5), (5.7) and (5.10) to find

$$
\begin{aligned}
\frac{d}{d t}\|v\|_{p}^{p}+\frac{\alpha}{2^{p+2}}\|v\|_{2 p-2}^{2 p-2} \leqslant & c_{1}\left(\|v\|_{p}^{p}+\|g(t, .)\|^{2}+\left\|\psi_{1}(t, .)\right\|_{\frac{p}{2}}^{\frac{p}{2}}+\left\|\psi_{3}(t, .)\right\|^{2}\right. \\
& \left.+\left|y\left(\vartheta_{t} \omega\right)\right|^{2 p-2}+\left|y\left(\vartheta_{t} \omega\right)\right|^{2}\right),
\end{aligned}
$$

where the letter $c_{1}=c_{1}\left(\alpha, p,\|\phi\|,\|\phi\|_{2 p-2},\left\|\mathcal{L}_{K} \phi\right\|,\left\|\psi_{2}\right\|_{\infty}\right)$. By employing Lemma 5.1(ii) with $\nu=0, r=2$ and $\epsilon=1$, along with $\omega$ replaced by $\vartheta_{-\tau} \omega$, it follows that for $\sigma \in[\tau-2, \tau]$,

$$
\begin{aligned}
& \left\|v\left(\sigma, \tau-t, \vartheta_{-\tau} \omega, v_{\tau-t}\right)\right\|_{p}^{p}+\frac{\alpha}{2^{p+2}} \int_{\tau-2}^{\tau}\|v(\xi)\|_{2 p-2}^{2 p-2} d \xi \\
& \leqslant 2 \int_{\tau-3}^{\tau}\left\|v\left(\xi, \tau-t, \vartheta_{-\tau} \omega, v_{\tau-t}\right)\right\|_{p}^{p} d \xi+3 c_{1} \int_{\tau-3}^{\tau}\left\|v\left(\xi, \tau-t, \vartheta_{-\tau} \omega, v_{\tau-t}\right)\right\|_{p}^{p} d \xi \\
& \quad+3 c_{1} \int_{\tau-3}^{\tau}\left(\|g(\xi, .)\|^{2}+\left\|\psi_{1}(\xi, .)\right\|_{\frac{p}{2}}^{\frac{p}{2}}+\left\|\psi_{3}(\xi, .)\right\|^{2}\right. \\
& \left.\quad+\left|y\left(\vartheta_{\xi-\tau} \omega\right)\right|^{2 p-2}+\left|y\left(\vartheta_{\xi-\tau} \omega\right)\right|^{2}\right) d \xi \\
& \leqslant c \int_{\tau-3}^{\tau}\left\|v\left(\xi, \tau-t, \vartheta_{-\tau} \omega, v_{\tau-t}\right)\right\|_{p}^{p} d \xi \\
& \quad+c \int_{\tau-3}^{\tau}\left(\|g(\xi, .)\|^{2}+\left\|\psi_{1}(\xi, .)\right\|_{\frac{p}{2}}^{\frac{p}{2}}+\left\|\psi_{3}(\xi, .)\right\|^{2}\right) d \xi \\
& \quad+c \int_{-3}^{0}\left(\left|y\left(\vartheta_{\xi} \omega\right)\right|^{2 p-2}+\left|y\left(\vartheta_{\xi} \omega\right)\right|^{2}\right) d \xi .
\end{aligned}
$$


By the continuity of $y\left(\vartheta_{t} \omega\right)$, in conjunction with $\psi_{1} \in L_{\text {loc }}^{p / 2}\left(\mathbf{R}, L^{p / 2}(\mathcal{O})\right)$ and $g, \psi_{3} \in$ $L_{\text {loc }}^{2}\left(\mathbf{R}, L^{2}(\mathcal{O})\right)$, it is clear that, for every $\tau \in \mathbf{R}$ and $\omega \in \Omega$,

$$
\begin{aligned}
& \int_{\tau-3}^{\tau}\left(\|g(\xi, .)\|^{2}+\left\|\psi_{1}(\xi, .)\right\|_{\frac{p}{2}}^{\frac{p}{2}}+\left\|\psi_{3}(\xi, .)\right\|^{2}\right) d \xi \\
& +\int_{-3}^{0}\left(\left|y\left(\vartheta_{\xi} \omega\right)\right|^{2 p-2}+\left|y\left(\vartheta_{\xi} \omega\right)\right|^{2}\right) d \xi<+\infty .
\end{aligned}
$$

On the other hand, by (4.2) in Lemma 4.1, there exists $T=T(\tau, \omega, D) \geqslant 3$, such that for all $t \geqslant T$,

$$
\int_{\tau-3}^{\tau}\left\|v\left(\xi, \tau-t, \vartheta_{-\tau} \omega, v_{\tau-t}\right)\right\|_{p}^{p} d \xi \leqslant c(1+C(\tau, \omega))
$$

Then the results are derived from (5.12)-(5.14).

Remark 3. From Lemma 5.2, we find that if the initial time goes to the negative infinite, the solution $u$ of problem $(3.1)-(3.3)$ belongs to $L_{\text {loc }}^{2 p-2}\left(\mathbf{R}, L^{2 p-2}(\mathcal{O})\right)$, which is crucial for us to prove the bound of solutions in the regular space $X_{0}^{s}$.

The following is concerned with a priori estimate of solutions in the Hilbert space $X_{0}^{s}$. Note that the conditions on the nonlinear function $F$ and the non-autonomous terms $g, \psi_{1}$ and $\psi_{3}$ are not added.

Lemma 5.3. Suppose that (3.4)-(3.8) and $\mathfrak{D}$ is defined by (3.17). Let $\tau \in$ $\mathbf{R}, \omega \in \Omega$ and $D=\{D(\tau, \omega): \tau \in \mathbf{R}, \omega \in \Omega\} \in \mathfrak{D}$ with $u_{\tau-t} \in D\left(\tau-t, \vartheta_{-t} \omega\right)$. Then there exist constants $M=M(\tau, \omega)>0$ and $T=T(\tau, \omega, D) \geqslant 3$ such that for all $t \geqslant T$, the solution $u$ of problem (3.13)-(3.15) satisfies

$$
\left\|v\left(\sigma, \tau-t, \vartheta_{-\tau} \omega, v_{\tau-t}\right)\right\|_{X_{0}^{s}}^{2} \leqslant M(\tau, \omega), \quad \sigma \in[\tau-1, \tau],
$$

where $v_{\tau-t}=u_{\tau-t}-z\left(\vartheta_{-t} \omega\right)$ and $M(\tau, \omega)>0$ is a constant depending $\alpha, p, \lambda_{1}$, $\|\phi\|,\|\phi\|_{2 p-2},\left\|\mathcal{L}_{K} \phi\right\|,\left\|\psi_{2}\right\|_{\infty}, C(\tau, \omega), \int_{\tau-3}^{\tau}\left\|\psi_{3}(\xi, .)\right\|^{2} d \xi, \int_{\tau-3}^{\tau}\left\|\psi_{1}(\xi, .)\right\|_{\frac{p}{2}}^{\frac{p}{2}} d \xi$ and $\int_{\tau-3}^{\tau}\|g(\xi, .)\|^{2} d \xi$

Proof. Using the test function $\mathcal{L}_{K} v$ in (3.13), we get

$$
\begin{aligned}
\frac{1}{2} \frac{d}{d t}\|v\|_{X_{0}^{s}}^{2}+\left\|\mathcal{L}_{K} v\right\|^{2}= & \left(F\left(t, x, v+z\left(\vartheta_{t} \omega\right)\right), \mathcal{L}_{K} v\right)+\left(g(t, x), \mathcal{L}_{K} v\right) \\
& +\left(z\left(\vartheta_{t} \omega\right), \mathcal{L}_{K} v\right)-\left(\mathcal{L}_{K} z\left(\vartheta_{t} \omega\right), \mathcal{L}_{K} v\right) \\
\leqslant & \frac{1}{2}\left\|\mathcal{L}_{K} v\right\|^{2}+c\|u\|_{2 p-2}^{2 p-2}+c\left\|\psi_{3}(t, .)\right\|^{2}+\|g(t, .)\|^{2} \\
& +c\left\|z\left(\vartheta_{t} \omega\right)\right\|^{2}+\left\|\mathcal{L}_{K} z\left(\vartheta_{t} \omega\right)\right\|^{2},
\end{aligned}
$$

which implies that

$$
\frac{d}{d t}\|v\|_{X_{0}^{s}}^{2} \leqslant c\left(\|v\|_{2 p-2}^{2 p-2}+\left\|\psi_{3}(t, .)\right\|^{2}+\|g(t, .)\|^{2}+\left|z\left(\vartheta_{t} \omega\right)\right|^{2}+\left|y\left(\vartheta_{t} \omega\right)\right|^{2 p-2}\right) .
$$

Utilizing Lemma 5.1(ii) with $\nu=0, r=1$ and $\epsilon=1$, we show for $\sigma \in[\tau-1, \tau]$,

$$
\begin{aligned}
& \left\|v\left(\sigma, \tau-t, \vartheta_{-\tau} \omega, v_{\tau-t}\right)\right\|_{X_{0}^{s}}^{2} \\
& \leqslant c \int_{\tau-2}^{\tau}\|v(\xi)\|_{2 p-2}^{2 p-2} d \xi+c \int_{\tau-3}^{\tau}\left(\left\|\psi_{3}(\xi, .)\right\|^{2}+\|g(\xi, .)\|^{2}\right) d \xi \\
& \quad+c \int_{\tau-3}^{\tau}\left(\left|z\left(\vartheta_{\xi-\tau} \omega\right)\right|^{2}+\left|y\left(\vartheta_{\xi-\tau} \omega\right)\right|^{2 p-2}\right) d \xi+2 \int_{\tau-2}^{\tau}\|v(\xi)\|_{X_{0}^{s}}^{2} d \xi .
\end{aligned}
$$


By (4.2) and (5.2), we know that there exist constants $T=T(\tau, \omega, D) \geqslant 3$ and $M_{1}(\tau, \omega)>1$ such that for all $t \geqslant T, \int_{\tau-2}^{\tau}\|v(\xi)\|_{2 p-2}^{2 p-2} d \xi \leqslant M_{1}(\tau, \omega)$ and $\int_{\tau-2}^{\tau}\|v(\xi)\|_{X_{0}^{s}}^{2} d \xi \leqslant M_{1}(\tau, \omega)$, respectively. And by (3.12), we have

$$
\int_{\tau-3}^{\tau}\left(\left|z\left(\vartheta_{\xi-\tau} \omega\right)\right|^{2}+\left|y\left(\vartheta_{\xi-\tau} \omega\right)\right|^{2 p-2}\right) d \xi \leqslant \varrho(\omega) \int_{\tau-3}^{\tau} e^{-\frac{1}{2} \lambda_{1}(\xi-\tau)} d \xi \leqslant 2 \varrho(\omega) \lambda_{1}^{-1} e^{\lambda_{1}} .
$$

Notice that $g, \psi_{3} \in L_{l o c}^{2}\left(\mathbf{R}, L^{2}(\mathcal{O})\right)$. Then we conclude the proof.

For $\tau \in \mathbf{R}, \omega \in \Omega$, given $v$ be the solution of the problem (3.13)-(3.15). Set $h=h(\tau, \omega) \geqslant 1$. Let $(v-h)_{+}=\max \{v-h, 0\}$ and $(v+h)_{-}=\min \{v+h, 0\}$. In order to derive the flattening condition, we need the following crucial estimate which shows that the $(2 p-2)$-truncation of solutions on a finite integral interval converges to zero if the initial time goes to the negative infinite and the eigenvalue of $\mathcal{L}_{K}$ is large enough.

Lemma 5.4. Suppose that that (3.4)-(3.8) hold. Given $\tau \in \mathbf{R}, \omega \in \Omega, D=$ $\{D(\tau, \omega): \tau \in \mathbf{R}, \omega \in \Omega\} \in \mathfrak{D}$ with $u_{\tau-t} \in D\left(\tau-t, \vartheta_{-t} \omega\right)$. Then for any $\varepsilon>0$, there exist constants $\tilde{h}=\tilde{h}(\tau, \omega, \varepsilon) \geqslant 1, N=N(\tau, \omega, \varepsilon) \in \mathbf{N}$ and $T=T(\tau, \omega, D) \geqslant 3$ such that the solution $v$ to the problem (3.13)-(3.15) satisfies that for all $t \geqslant T$ and $k \geqslant N$,

$$
\int_{\tau-1}^{\tau} e^{\lambda_{k+1}(\xi-\tau)} \int_{\tilde{\mathcal{O}}}\left|v\left(\xi, \tau-t, \vartheta_{-\tau} \omega, \tau-t, v_{\tau-t}\right)\right|^{2 p-2} d x d \xi \leqslant c \varepsilon
$$

where $\tilde{\mathcal{O}}=\left\{x \in \mathcal{O}:\left|v\left(\xi, \tau-t, \vartheta_{-\tau} \omega, v_{\tau-t}\right)\right| \geqslant \tilde{h}, \xi \in[\tau-1, \tau]\right\}$ and $\lambda_{k+1}$ is the eigenvalue of non-local operator $\mathcal{L}_{K}, c>0$ is a deterministic constant independent of $\tilde{h}, \tau$ and $\omega, v_{\tau-t}=u_{\tau-t}-z\left(\vartheta_{-t} \omega\right)$ and $p \geqslant 2$.

Proof. It is clear that (5.15) holds true for $p=2$, by means of (4.14). Without loss of generality, we let $p>2$ in what follows. Given $\tau \in \mathbf{R}$ and $\omega \in \Omega$ fixed, we first replace $\omega$ by $\vartheta_{-\tau} \omega$ in (3.13) to see that $v=v(\xi)=v\left(\xi, \tau-t, \vartheta_{-\tau} \omega, v_{\tau-t}\right), \xi \in[\tau-1, \tau]$ is a solution to the stochastic differential equation at the sample $\vartheta_{-\tau} \omega$ with the initial datum $v_{\tau-t}$ at the initial time $\tau-t$ :

$$
\frac{d v}{d \xi}+\mathcal{L}_{K} v(x)=F\left(\xi, x, v+z\left(\vartheta_{\xi-\tau} \omega\right)\right)+g(\xi, x)+z\left(\vartheta_{\xi-\tau} \omega\right)-\mathcal{L}_{K} z\left(\vartheta_{\xi-\tau} \omega\right)
$$

with the initial datum $v_{\tau-t}=u_{\tau-t}-z\left(\vartheta_{-t} \omega\right)$. For every $\tau \in \mathbf{R}, \omega \in \Omega$ and all $\xi \in[-1,0]$, let the large constant

$$
h=h(\tau, \omega)>\aleph(\omega)=\sup _{\xi \in[-1,0]}\left\{\|\phi\|_{\infty}\left|y\left(\vartheta_{\xi} \omega\right)\right|\right\},
$$

where $\aleph(\omega)$ is finite by the continuity of $y\left(\vartheta_{\xi} \omega\right)$ and $\phi \in L^{\infty}$. Indeed, by (3.12), we have $\left|y\left(\vartheta_{\xi} \omega\right)\right| \leqslant \sqrt{\varrho(\omega)} e^{\frac{1}{4} \lambda_{1}}<\infty$, for any $\xi \in[-1,0]$.

Using the test function $\left|(v-h)_{+}\right|^{p-2}(v-h)_{+}=(v-h)_{+}^{p-1}$ in (5.16), we get that, for every $\xi \in[\tau-1, \tau]$,

$$
\begin{aligned}
& \frac{1}{p} \frac{d}{d \xi} \int_{\mathcal{O}}(v-h)_{+}^{p} d x+\left(\mathcal{L}_{K} v(x),(v-h)_{+}^{p-1}\right)=\int_{\mathcal{O}} F(\xi, x, u)(v-h)_{+}^{p-1} d x \\
& \left.\quad+\int_{\mathcal{O}}\left(g(\xi, x)+z\left(\vartheta_{\xi-\tau} \omega\right)\right)(v-h)_{+}^{p-1}\right) d x-\left(\mathcal{L}_{K} z\left(\vartheta_{\xi-\tau} \omega\right),(v-h)_{+}^{p-1}\right) .
\end{aligned}
$$


Recall from Lemma 6.3 in [2] that, for any $d \geqslant 2$, there exists a constant $c_{N, d}>0$ such that

$$
\left(|x|^{d-2} x-|y|^{d-2} y\right) \cdot(x-y) \geqslant c_{N, d}|x-y|^{d},
$$

where "." is the inner product in $\mathbf{R}^{N}$.

We begin with the estimate of the second term on the left of (5.18). Indeed, we have

$$
\begin{aligned}
& \left(\mathcal{L}_{K} v(x),(v-h)_{+}^{p-1}\right) \\
& =\int_{\mathbf{R}^{2 N}}(v(x)-v(y))\left((v(x)-h)_{+}^{p-1}-(v(y)-h)_{+}^{p-1}\right) K(x-y) d x d y .
\end{aligned}
$$

Let $\mathbf{R}^{2 N}=S_{1} \cup S_{2} \cup S_{3} \cup S_{4}$, where

$$
\begin{aligned}
& S_{1} \triangleq\left\{(x, y) \in \mathbf{R}^{2 N}: v(x) \geqslant h, v(y) \geqslant h, x, y \in \mathbf{R}^{N}\right\} ; \\
& S_{2} \triangleq\left\{(x, y) \in \mathbf{R}^{2 N}: v(x) \leqslant h, v(y) \leqslant h, x, y \in \mathbf{R}^{N}\right\} ; \\
& S_{3} \triangleq\left\{(x, y) \in \mathbf{R}^{2 N}: v(x) \geqslant h, v(y) \leqslant h, x, y \in \mathbf{R}^{N}\right\} ; \\
& S_{4} \triangleq\left\{(x, y) \in \mathbf{R}^{2 N}: v(x) \leqslant h, v(y) \geqslant h, x, y \in \mathbf{R}^{N}\right\} .
\end{aligned}
$$

Then by (5.19), it is clear that

$$
\begin{aligned}
& \int_{S_{1}}(v(x)-v(y))\left((v(x)-h)_{+}^{p-1}-(v(y)-h)_{+}^{p-1}\right) K(x-y) d x d y \\
& =\int_{S_{1}}\left((v(x)-h)_{+}-(v(y)-h)_{+}\right)\left((v(x)-h)_{+}^{p-1}-(v(y)-h)_{+}^{p-1}\right) \\
& \quad \cdot K(x-y) d x d y \\
& \geqslant \int_{S_{1}}\left|(v(x)-h)_{+}-(v(y)-h)_{+}\right|^{p} K(x-y) d x d y \geqslant 0 .
\end{aligned}
$$

Since $(v(x)-h)_{+}=(v(y)-h)_{+}=0$ on $S_{2}$, then

$$
\int_{S_{2}}(v(x)-v(y))\left((v(x)-h)_{+}^{p-1}-(v(y)-h)_{+}^{p-1}\right) K(x-y) d x d y=0 .
$$

Noticing that $(v(x)-v(y))\left((v(x)-h)_{+}^{p-1}-(v(y)-h)_{+}^{p-1}\right)=((v(x)-h)-(v(y)-$ $h))(v(x)-h)_{+}^{p-1} \geqslant 0$ on $S_{3}$, we have

$$
\int_{S_{3}}(v(x)-v(y))\left((v(x)-h)_{+}^{p-1}-(v(y)-h)_{+}^{p-1}\right) K(x-y) d x d y \geqslant 0,
$$

and similarly, we also have

$$
\int_{S_{4}}(v(x)-v(y))\left((v(x)-h)_{+}^{p-1}-(v(y)-h)_{+}^{p-1}\right) K(x-y) d x d y \geqslant 0 .
$$

From (5.20)-(5.24), we thereby obtain that

$$
\left(\mathcal{L}_{K} v(x),(v-h)_{+}^{p-1}\right) \geqslant 0 .
$$

We then estimate the nonlinearity in (5.18). For convenience, we write $\mathcal{O}_{h}=$ $\left\{x \in \mathcal{O}:\left|v\left(\xi, \tau-t, \vartheta_{-\tau} \omega, v_{\tau-t}\right)\right| \geqslant h\right\}$ with $\xi \in[\tau-1, \tau]$.

Thanks to $v \geqslant h$, in conjunction with (5.17), it follows that

$$
u(\xi)=v(\xi)+z\left(\vartheta_{\xi-\tau} \omega\right) \geqslant v(\xi)-\aleph(\omega)>v(\xi)-h \geqslant 0,
$$


for $\xi \in[\tau-1, \tau]$, and consequently by (3.4), we have

$$
F(\xi, x, u) \leqslant-\alpha u^{p-1}+\frac{\psi_{1}(x)}{u}, \quad x \in \mathcal{O}_{h} .
$$

By the inequality $|u|^{p-1} \geqslant 2^{2-p}|v|^{p-1}-\left|z\left(\vartheta_{\xi-\tau} \omega\right)\right|^{p-1}$, the result (5.26) is rephrased

$$
F(\xi, x, u) \leqslant-\frac{\alpha}{2^{p-2}} v^{p-1}+\left|z\left(\vartheta_{\xi-\tau} \omega\right)\right|^{p-1}+\frac{\psi_{1}(x)}{v-h}, \quad x \in \mathcal{O}_{h} .
$$

Note that

$$
v^{p-1} \geqslant v^{p-2}(v-h) \geqslant h^{p-2}(v-h), \quad x \in \mathcal{O}_{h} .
$$

Then by a splitting of the term $-\frac{\alpha}{2^{p-2}} v^{p-1}$ into two $\frac{1}{2}$-terms, with the one of them using (5.28), we get

$$
\begin{aligned}
F(\xi, x, u) \leqslant & -\frac{\alpha}{2^{p-1}} h^{p-2}(v-h)_{+}-\frac{\alpha}{2^{p-1}} v^{p-1} \\
& +\left|z\left(\vartheta_{\xi-\tau} \omega\right)\right|^{p-1}+\frac{\psi_{1}(x)}{(v-h)_{+}}, \quad x \in \mathcal{O}_{h},
\end{aligned}
$$

by which, the nonlinearity in (5.16) is estimated as

$$
\begin{aligned}
\int_{\mathcal{O}} F(\xi, x, u)(v-h)_{+}^{p-1} d x & \leqslant-\frac{\alpha}{2^{p-1}} h^{p-2} \int_{\mathcal{O}}(v-h)_{+}^{p} d x-\frac{\alpha}{2^{p-1}} \int_{\mathcal{O}} v^{p-1}(v-h)_{+}^{p-1} d x \\
& +\int_{\mathcal{O}}\left|z\left(\vartheta_{\xi-\tau} \omega\right)\right|^{p-1}(v-h)_{+}^{p-1} d x+\int_{\mathcal{O}} \psi_{1}(x)(v-h)_{+}^{p-2} d x
\end{aligned}
$$

where we note that

$$
\frac{\alpha}{2^{p-1}} \int_{\mathcal{O}} v^{p-1}(v-h)_{+}^{p-1} d x \geqslant \frac{\alpha}{2^{p-1}} \int_{\mathcal{O}}(v-h)_{+}^{2 p-2} d x,
$$

and by Young inequality and the condition $\phi \in L^{2 p-2}$, we get

$$
\begin{aligned}
& \int_{\mathcal{O}}\left|z\left(\vartheta_{\xi-\tau} \omega\right)\right|^{p-1}(v-h)_{+}^{p-1} d x \\
& \leqslant \frac{\alpha}{2^{p}} \int_{\mathcal{O}}(v-h)_{+}^{2 p-2} d x+c\|\phi\|_{2 p-2}^{2 p-2}\left|y\left(\vartheta_{\xi-\tau} \omega\right)\right|^{2 p-2},
\end{aligned}
$$

and by the condition $\psi_{1} \in L_{\text {loc }}^{\frac{p}{2}}\left(\mathbf{R}, L^{\frac{p}{2}}\right)$ and the assumption $h \geqslant 1$, we have

$$
\begin{aligned}
\int_{\mathcal{O}} \psi_{1}(\xi, x)(v-h)_{+}^{p-2} d x & \leqslant \frac{\alpha}{2^{p}} h^{p-2} \int_{\mathcal{O}}(v-h)_{+}^{p} d x+\left(\frac{\alpha}{2^{p}} h^{p-2}\right)^{-\frac{p-2}{2}}\left\|\psi_{1}(\xi, .)\right\|_{\frac{p}{2}}^{\frac{p}{2}} \\
& \leqslant \frac{\alpha}{2^{p}} h^{p-2} \int_{\mathcal{O}}(v-h)_{+}^{p} d x+\left(\frac{\alpha}{2^{p}}\right)^{-\frac{p-2}{2}}\left\|\psi_{1}(\xi, .)\right\|_{\frac{p}{2}}^{\frac{p}{2}}
\end{aligned}
$$

where $c=c(p, \alpha)$ independent of $h, \tau$ and $\omega$. Combine (5.31)-(5.33) into the right side of (5.30) to yield

$$
\begin{aligned}
\int_{\mathcal{O}} F(\xi, x, u)(v-h)_{+}^{p-1} d x \leqslant & -\frac{\alpha}{2^{p}} h^{p-2} \int_{\mathcal{O}}(v-h)_{+}^{p} d x-\frac{\alpha}{2^{p}} \int_{\mathcal{O}}(v-h)_{+}^{2 p-2} d x \\
& +c\left(\left|y\left(\vartheta_{\xi-\tau} \omega\right)\right|^{2 p-2}+\left\|\psi_{1}(\xi, .)\right\|_{\frac{p}{2}}^{\frac{p}{2}}\right),
\end{aligned}
$$

where $c=c\left(\alpha, p,\|\phi\|,\|\phi\|_{2 p-2}\right)$. This completes the estimate of the nonlinear term in (5.18). 
The second term on the right hand side of (5.16) is bounded by

$$
\begin{aligned}
& \int_{\mathbf{R}^{N}}\left(g(\xi, x)+z\left(\vartheta_{\xi-\tau} \omega\right)\right)(v(\xi)-h)_{+}^{p-1} d x \\
& \leqslant \frac{\alpha}{2^{p+2}} \int_{\mathcal{O}}(v-h)_{+}^{2 p-2} d x+c\|g(\xi, .)\|^{2}+c\left|y\left(\vartheta_{\xi-\tau} \omega\right)\right|^{2},
\end{aligned}
$$

where $c=c(\alpha, p,\|\phi\|)$ For the last term in (5.16), thanks to $\mathcal{L}_{K} \phi \in L^{2}(\mathcal{O})$, we have

$$
\begin{aligned}
& \left(\mathcal{L}_{K} z\left(\vartheta_{\xi-\tau} \omega\right),(v-h)_{+}^{p-1}\right)=\int_{\mathbf{R}^{2 N}} y\left(\vartheta_{\xi-\tau} \omega\right)(\phi(x)-\phi(y))\left((v(x)-h)_{+}^{p-1}\right. \\
& \left.\left.\quad-(v(y)-h)_{+}^{p-1}\right)\right) K(x-y) d x d y \\
& =\int_{\mathbf{R}^{2 N}} y\left(\vartheta_{\xi-\tau} \omega\right)(\phi(x)-\phi(y))(v(x)-h)_{+}^{p-1} K(x-y) d x d y \\
& \quad-\int_{\mathbf{R}^{2 N}} y\left(\vartheta_{\xi-\tau} \omega\right)(\phi(x)-\phi(y))(v(y)-h)_{+}^{p-1} K(x-y) d x d y .
\end{aligned}
$$

By Hölder inequality,

$$
\begin{aligned}
& \int_{\mathbf{R}^{2 N}}\left(y\left(\vartheta_{\xi-\tau} \omega\right)(\phi(x)-\phi(y))(v(x)-h)_{+}^{p-1} K(x-y) d x d y\right. \\
& \leqslant\left\{\int_{\mathbf{R}^{N}}\left(\int_{\mathbf{R}^{N}} y\left(\vartheta_{\xi-\tau} \omega\right)(\phi(x)-\phi(y)) K(x-y) d y\right)^{2} d x\right\}^{\frac{1}{2}} \\
& \quad \cdot\left\{\int_{\mathbf{R}^{N}}(v(x)-h)_{+}^{2 p-2} d x\right\}^{\frac{1}{2}} \\
& \leqslant \frac{\alpha}{2^{p+3}} \int_{\mathcal{O}}(v-h)_{+}^{2 p-2} d x+c\left|y\left(\vartheta_{\xi-\tau}\right)\right|^{2}\left\|\mathcal{L}_{K} \phi\right\|^{2},
\end{aligned}
$$

and for the second term in (5.36), we have a similar estimate. Then by (5.36) and (5.37) we get

$$
\left(\mathcal{L}_{K} z\left(\vartheta_{\xi-\tau} \omega\right),(v-h)_{+}^{p-1}\right) \leqslant \frac{\alpha}{2^{p+2}} \int_{\mathcal{O}}(v-h)_{+}^{2 p-2} d x+c\left|y\left(\vartheta_{\xi-\tau}\right)\right|^{2},
$$

where $c=c\left(\alpha, p,\left\|\mathcal{L}_{K} \phi\right\|\right)$.

Therefore, by a combination of (5.25), (5.34), (5.35) and (5.38) into (5.18), we finally find that

$$
\begin{aligned}
& \frac{d}{d \xi} \int_{\mathcal{O}}(v-h)_{+}^{p} d x+\chi(h) \int_{\mathcal{O}}(v-h)_{+}^{p}+\frac{\alpha}{2^{p+1}} \int_{\mathcal{O}}(v-h)_{+}^{2 p-2} d x \\
& \leqslant c\left(\left|y\left(\vartheta_{\xi-\tau} \omega\right)\right|^{2}+\left|y\left(\vartheta_{\xi-\tau} \omega\right)\right|^{2 p-2}+\|g(\xi, .)\|^{2}+\left\|\psi_{1}(\xi, .)\right\|_{\frac{p}{2}}^{\frac{p}{2}}\right),
\end{aligned}
$$

where $\chi(h)=\frac{\alpha}{2^{p-1}} h^{p-2} \uparrow \infty$ as $h \rightarrow \infty$, and the positive constant $c$ is independent of $h$. By employing Gronwall lemma over the interval $[\tau-1, \tau]$, we deduce that

$$
\begin{aligned}
\int_{\tau-1}^{\tau} e^{\chi(h)(\xi-\tau)} \int_{\mathcal{O}} & (v(\xi)-h)_{+}^{2 p-2} d x d \xi \leqslant c e^{-\chi(h)}\|v(\tau-1)\|_{p}^{p} \\
& +c \int_{\tau-1}^{\tau} e^{\chi(h)(\xi-\tau)}\left(\left|y\left(\vartheta_{\xi-\tau} \omega\right)\right|^{2}+\left|y\left(\vartheta_{\xi-\tau} \omega\right)\right|^{2 p-2}\right) d \xi \\
& +c \int_{\tau-1}^{\tau} e^{\chi(h)(\xi-\tau)}\left(\|g(\xi, .)\|^{2}+\left\|\psi_{1}(\xi, .)\right\|_{\frac{p}{2}}^{\frac{p}{2}}\right) d \xi .
\end{aligned}
$$


By (5.1), there exists a constant $T=T(\tau, \omega, D) \geqslant 3$, such that for all $t \geqslant T$, $\|v(\xi)\|_{p}^{p} \leqslant M(\tau, \omega)$ for $\xi \in[\tau-2, \tau]$, and thereby we have

$$
c e^{-\chi(h)}\|v(\tau-1)\|_{p}^{p} \leqslant c M(\tau, \omega) e^{-\chi(h)} \leqslant \frac{\varepsilon}{4},
$$

for any $h \geqslant h_{1}$ with $h_{1}=h_{1}(\tau, \omega, \varepsilon)$. By (3.12), we get

$$
\begin{aligned}
c \int_{\tau-1}^{\tau} e^{\chi(h)(\xi-\tau)}\left(\left|y\left(\vartheta_{\xi-\tau} \omega\right)\right|^{2}+\left|y\left(\vartheta_{\xi-\tau} \omega\right)\right|^{2 p-2}\right) d \xi & \leqslant c \varrho(\omega) \int_{\tau-1}^{\tau} e^{\left(\chi(h)-\frac{1}{2} \lambda_{1}\right)(\xi-\tau)} d \xi \\
& \leqslant \frac{c \varrho(\omega)}{\chi(h)-\frac{1}{2} \lambda_{1}} \leqslant \frac{\varepsilon}{4},
\end{aligned}
$$

for any $h \geqslant h_{2}$ with some $h_{2}=h_{2}(\tau, \omega, \varepsilon)$. We next cope with the third term on the right hand side of (5.40). Indeed, choosing $M$ large enough such that $\chi(h)>\lambda_{1}$, and taking $\varsigma \in(0,1)$ small, we have

$$
\begin{aligned}
& \int_{\tau-1}^{\tau} e^{\chi(h)(\xi-\tau)}\|g(\xi, .)\|^{2} d \xi=\int_{\tau-1}^{\tau-\varsigma} e^{\chi(h)(\xi-\tau)}\|g(\xi, .)\|^{2} d \xi+\int_{\tau-\varsigma}^{\tau} e^{\chi(h)(\xi-\tau)}\|g(\xi, .)\|^{2} d \xi \\
& =e^{-\chi(h) \tau} \int_{\tau-1}^{\tau-\varsigma} e^{\left(\chi(h)-\lambda_{1}\right) \xi} e^{\lambda_{1} \xi}\|g(\xi, .)\|^{2} d \xi+\int_{\tau-\varsigma}^{\tau} e^{\chi(h)(\xi-\tau)}\|g(\xi, .)\|^{2} d \xi \\
& \leqslant e^{-\chi(h) \varsigma} e^{\lambda_{1}(\varsigma-\tau)} \int_{-\infty}^{\tau} e^{\lambda_{1} \xi}\|g(\xi, .)\|^{2} d \xi+\int_{\tau-\varsigma}^{\tau}\|g(\xi, .)\|^{2} d \xi .
\end{aligned}
$$

By (3.8), $\int_{-\infty}^{\tau} e^{\lambda_{1} \xi}\|g(\xi, .)\|^{2} d \xi$ is bounded, therefore we have

$$
e^{-\chi(h) \varsigma} e^{\lambda_{1}(\varsigma-\tau)} \int_{-\infty}^{\tau} e^{\lambda_{1} \xi}\|g(\xi, .)\|^{2} d \xi \rightarrow 0
$$

as $h \rightarrow \infty$. On the other hand, since $g \in L_{\text {loc }}^{2}\left(\mathbf{R}, L^{2}(\mathcal{O})\right)$, we can choose $\varsigma$ small enough such that $\int_{\tau-\varsigma}^{\tau}\|g(\xi, .)\|^{2} d \xi \rightarrow 0$. Based on this analysis, we hence deduce that

$$
c \int_{\tau-1}^{\tau} e^{\chi(h)(\xi-\tau)}\|g(\xi, .)\|^{2} d \xi \leqslant \frac{\varepsilon}{8}
$$

for any $h \geqslant h_{3}$ with some $h_{3}=h_{3}(\tau, \varepsilon)>\lambda_{1}$. By a similar argument, using (3.8) we have

$$
c \int_{\tau-1}^{\tau} e^{\chi(h)(\xi-\tau)}\left\|\psi_{1}(\xi, .)\right\|_{\frac{p}{2}}^{\frac{p}{2}} d \xi \leqslant \frac{\varepsilon}{8}
$$

for any $h \geqslant h_{4}$ with some $h_{4}=h_{4}(\tau, \varepsilon)>\lambda_{1}$.

Put $h_{0}=\max \left\{h_{1}, h_{2}, h_{3}, h_{4}\right\} \geqslant \lambda_{1}$. By plugging (5.41)-(5.43) into (5.40), we finally get that

$$
\int_{\tau-1}^{\tau} e^{\chi(h)(\xi-\tau)} \int_{\mathcal{O}}(v(\xi)-h)_{+}^{2 p-2} d x d \xi \leqslant \varepsilon,
$$

for any $h \geqslant h_{0}$. Note that the eigenvalue sequence $\left\{\lambda_{k}\right\}_{k \in \mathbf{N}}$ of the operator $\mathcal{L}_{K}$ is increasing to the positive infinity. Then we can choose $\lambda_{k+1}>\chi(h) \geqslant \chi\left(h_{0}\right)$ for any $k \geqslant N$ with some $N=N(\tau, \omega, \varepsilon)$. Then (5.45) is rewritten as

$$
\int_{\tau-1}^{\tau} e^{\lambda_{k+1}(\xi-\tau)} \int_{\mathcal{O}_{h}}(v(\xi)-h)_{+}^{2 p-2} d x d \xi \leqslant \varepsilon
$$


for any $h \geqslant h_{0}$ and $k \geqslant N$. Noticing that $v \leqslant 2(v-h)_{+}$for $v \geqslant 2 h$, it follows from (5.46) that

$$
\begin{aligned}
\int_{\tau-1}^{\tau} e^{\lambda_{k+1}(\xi-\tau)} \int_{\mathcal{O}_{2 h}}|v(\xi)|^{2 p-2} d x d \xi & \leqslant \int_{\tau-1}^{\tau} e^{\lambda_{k+1}(\xi-\tau)} \int_{\mathcal{O}_{h}}(v(\xi)-h)_{+}^{2 p-2} d x d \xi \\
& \leqslant 2^{2 p-2} \varepsilon,
\end{aligned}
$$

where $\mathcal{O}_{2 h} \triangleq\left\{x \in \mathcal{O}: v\left(\xi, \tau-t, \vartheta_{-\tau} \omega, v_{\tau-t}\right) \geqslant 2 h\right\} \subseteq \mathcal{O}_{h}$ with $\xi \in[\tau-1, \tau]$. By a whole parallel way, using the test function $\left|(v+h)_{-}\right|^{p-2}(v+h)_{-}$, we can show that there exist $\tilde{h}_{0}=\tilde{h}_{0}(\tau, \omega, \varepsilon)>1, T_{1}=T_{1}(\tau, \omega, D) \geqslant 3$ and $N_{1}=N_{1}(\tau, \omega, \varepsilon) \in \mathbf{N}$ such that for all $h>\tilde{h}_{0}, t \geqslant T_{1}$ and $k \geqslant N_{1}$,

$$
\int_{\tau-1}^{\tau} e^{\lambda_{k+1}(\xi-\tau)} \int_{\mathcal{O}_{-2 h}}|v(\xi)|^{2 p-2} d x d \xi \leqslant 2^{2 p-2} \varepsilon,
$$

where $\mathcal{O}_{-2 h} \triangleq\left\{x \in \mathcal{O}: v\left(\xi, \tau-t, \vartheta_{-\tau} \omega, v_{\tau-t}\right) \leqslant-2 h\right\}$ with $\xi \in[\tau-1, \tau]$. Then the proof is concluded by (5.47) and (5.48).

Remark 4. The non-autonomous terms $\psi_{1}$ and $g$ have weaker assumptions $\psi_{1} \in L_{\text {loc }}^{\frac{p}{2}}\left(\mathbf{R}, L^{\frac{p}{2}}(\mathcal{O})\right)$ and $g \in L^{2}\left(\mathbf{R}, L^{2}(\mathcal{O})\right)$ than $\psi_{1} \in L^{\infty}\left(\mathbf{R}, L^{\frac{p}{2}}(\mathcal{O})\right)$ and $g \in$ $L^{\infty}\left(\mathbf{R}, L^{2}(\mathcal{O})\right)$ in [13]. On the other hand, the assumption that $\phi \in L^{\infty}(\mathcal{O})$ is separately used in the process of deriving this truncation estimate of solutions which is necessary to prove the flattening condition in what follows.

5.2. Spectrum splitting method and flattening condition. To obtain the regularity of pullback attractor in $X_{0}^{s}$, we need to prove the flattening condition of the cocycle defined by (3.16) in $X_{0}^{s}$, which is derived by the spectrum splitting technique. Thanks to Lemma 2.2 (ii), the non-local operator $\mathcal{L}_{K}$ has a family of eigenvectors $\left\{e_{j}\right\}_{j \in \mathbf{N}}$ such that $\left\{e_{j}\right\}_{j \in \mathbf{N}}$ is an orthonormal basis of both $L^{2}(\mathcal{O})$ and $X_{0}^{s}$ with $\left\|e_{j}\right\|=1$ for every $j \in \mathbf{N}$, and the corresponding eigenvalues $\lambda_{j} \uparrow \infty$ as $j \rightarrow \infty$. Thus for any $v \in X_{0}^{s}$, we have $v=\sum_{j=1}^{\infty} c_{j} e_{j}$ for some $c_{j} \in \mathbf{R}, j \in \mathbf{N}$. Then the non-local operator $\mathcal{L}_{K}$ can be formulated as

$$
\mathcal{L}_{K} v=\sum_{j=1}^{\infty} c_{j} \mathcal{L}_{K} e_{j}=\sum_{j=1}^{\infty} c_{j} \lambda_{j} e_{j}, \quad v \in X_{0}^{s}
$$

and therefore $\left\|\mathcal{L}_{K} v\right\|^{2}=\sum_{j=1}^{\infty}\left(c_{j} \lambda_{j}\right)^{2}$. We make a direct sum decomposition of the space $X_{0}^{s}$ by

$$
X_{0}^{s}=\operatorname{span}\left\{e_{1}, e_{2}, \ldots, e_{k}\right\} \oplus\left(\operatorname{span}\left\{e_{1}, e_{2}, \ldots, e_{k}\right\}\right)^{\perp}=\operatorname{span}\left\{e_{1}, e_{2}, \ldots, e_{k}\right\} \oplus \mathbf{P}_{k+1},
$$

where $\mathbf{P}_{k+1}=\left\{u \in X_{0}^{s}:\left(u, e_{j}\right)_{X_{0}^{s}}=0, \forall j=1,2, \ldots, k\right\}$. It is noted that the orthogonal $\perp$ is calculated with respect to the scalar product of $X_{0}^{s}$ which is defined by (2.6). Thus, given any $v \in X_{0}^{s}$, we write $v=v_{1}+v_{2}$ with $v_{2} \in \mathbf{P}_{k+1}$. Define a project operator $P_{k}$ by

$$
P_{k}: X_{0}^{s} \rightarrow \operatorname{span}\left\{e_{1}, e_{2}, \ldots, e_{k}\right\}
$$

Then $P_{k} X_{0}^{s}=\operatorname{span}\left\{e_{1}, e_{2}, \ldots, e_{k}\right\}$ and $\left(I-P_{k}\right) X_{0}^{s}=\mathbf{P}_{k+1}$, and therefore, $v=$ $v_{1}+v_{2}=P_{k} v+\left(I-P_{k}\right) v$ for any $v \in X_{0}^{s}$, such that

$$
v_{1} \triangleq P_{k} v=\sum_{j=1}^{k} c_{j} \lambda_{j} e_{j}, \quad v_{2} \triangleq\left(I-P_{k}\right) v=\sum_{j=k+1}^{\infty} c_{j} \lambda_{j} e_{j} .
$$


The following lemma is concerned with the flattening condition of solutions.

Lemma 5.5. Let $s \in(0,1)$ and $N>2 s$. Suppose that (3.4)-(3.8) hold and $\mathfrak{D}$ is defined by (3.17). Let $\tau \in \mathbf{R}, \omega \in \Omega$ and $D=\{D(\tau, \omega): \tau \in \mathbf{R}, \omega \in \Omega\} \in \mathfrak{D}$ with $u_{\tau-t} \in D\left(\tau-t, \vartheta_{-t} \omega\right)$. Then for every $\varepsilon>0$, there exist $T=T(\tau, \omega, D, \varepsilon) \geqslant 3$ and $N_{0}=N_{0}(\tau, \omega, \varepsilon) \in \mathbf{N}$ such that for all $t \geqslant T$ and $k \geqslant N_{0}$, the solution $u$ of problem (3.1)-(3.3) satisfies

$$
\left\|\left(I-P_{k}\right) u\left(\tau, \tau-t, \vartheta_{-\tau} \omega, u_{\tau-t}\right)\right\|_{X_{0}^{s}} \leqslant \varepsilon .
$$

Proof. From (3.13), tested with $\mathcal{L}_{K} v_{2}$, we obtain that

$$
\begin{aligned}
& \int_{\mathbf{R}^{2 N}}\left(v_{t}(x)-v_{t}(y)\right)\left(v_{2}(x)-v_{2}(y)\right) K(x-y) d x d y+\left(\mathcal{L}_{K} v, \mathcal{L}_{K} v_{2}\right) \\
& =\int_{\mathcal{O}} F\left(t, x, v+z\left(\vartheta_{t} \omega\right)\right) \mathcal{L}_{K} v_{2} d x+\int_{\mathcal{O}} g(t, x) \mathcal{L}_{K} v_{2} d x \\
& \quad+\int_{\mathcal{O}} z\left(\vartheta_{t} \omega\right) \mathcal{L}_{K} v_{2} d x-\left(\mathcal{L}_{K} z\left(\vartheta_{t} \omega\right), \mathcal{L}_{K} v_{2}\right) .
\end{aligned}
$$

By the $\perp$ relation of $v_{1}$ and $v_{2}$ in $X_{0}^{s}$, we deduce that

$$
\int_{\mathbf{R}^{2 N}}\left(v_{t}(x)-v_{t}(y)\right)\left(v_{2}(x)-v_{2}(y)\right) K(x-y) d x d y=\frac{1}{2} \frac{d}{d t}\left\|v_{2}\right\|_{X_{0}^{s}} .
$$

Thanks to (5.51), we have

$$
\mathcal{L}_{K} v_{2}=\sum_{j=k+1}^{\infty} c_{j} \lambda_{j} e_{j}
$$

Then along with (5.49), it produces that

$$
\left(\mathcal{L}_{K} v, \mathcal{L}_{K} v_{2}\right)=\left(\sum_{j=1}^{\infty} c_{j} \lambda_{j} e_{j}, \sum_{j=k+1}^{\infty} c_{j} \lambda_{j} e_{j}\right)=\sum_{j=k+1}^{\infty} c_{j}^{2}\left(\lambda_{j}\right)^{2}=\left\|\mathcal{L}_{K} v_{2}\right\|^{2} .
$$

By plugging (5.53) and (5.54) into (5.52), we obtain

$$
\begin{aligned}
\frac{1}{2} \frac{d}{d t}\left\|v_{2}\right\|_{X_{0}^{s}}+\left\|\mathcal{L}_{K} v_{2}\right\|^{2}= & \int_{\mathcal{O}} F\left(t, x, v+z\left(\vartheta_{t} \omega\right)\right) \mathcal{L}_{K} v_{2} d x+\int_{\mathcal{O}} g(t, x) \mathcal{L}_{K} v_{2} d x \\
& +\int_{\mathcal{O}} z\left(\vartheta_{t} \omega\right) \mathcal{L}_{K} v_{2} d x-\left(\mathcal{L}_{K} z\left(\vartheta_{t} \omega\right), \mathcal{L}_{K} v_{2}\right) .
\end{aligned}
$$

We now estimate every term on the right hand side of (5.55). For the nonlinearity term, by (3.5) we get

$$
\begin{aligned}
& \int_{\mathcal{O}} F\left(t, x, v+z\left(\vartheta_{t} \omega\right)\right) \mathcal{L}_{K} v_{2} d x \leqslant \frac{1}{6}\left\|\mathcal{L}_{K} v_{2}\right\|^{2}+\frac{3}{2} \int_{\mathcal{O}}\left|F\left(t, x, v+z\left(\vartheta_{t} \omega\right)\right)\right|^{2} d x \\
& \left.\leqslant \frac{1}{6}\left\|\mathcal{L}_{K} v_{2}\right\|^{2}+3\left\|\psi_{2}\right\|_{\infty}^{2} \int_{\mathcal{O}} \mid v+z\left(\vartheta_{t} \omega\right)\right)\left.\right|^{2 p-2} d x+3\left\|\psi_{3}(t, .)\right\|^{2} \\
& \leqslant \frac{1}{6}\left\|\mathcal{L}_{K} v_{2}\right\|^{2}+c\left\|\left|v\left\|_{2 p-2}^{2 p-2}+c\left|y\left(\vartheta_{t} \omega\right)\right|^{2 p-2}+c\right\| \psi_{3}(t, .) \|^{2} .\right.\right.
\end{aligned}
$$

It is easy to calculate that

$$
\begin{aligned}
& \int_{\mathcal{O}} g(t, x) \mathcal{L}_{K} v_{2} d x+\int_{\mathcal{O}} z\left(\vartheta_{t} \omega\right) \mathcal{L}_{K} v_{2} d x \\
& \leqslant \frac{1}{6}\left\|\mathcal{L}_{K} v_{2}\right\|^{2}+c\left(\|g(t, .)\|^{2}+\|\phi\|^{2}\left|y\left(\vartheta_{t} \omega\right)\right|^{2}\right) .
\end{aligned}
$$


Since $\mathcal{L}_{K} \phi \in L^{2}(\mathcal{O})$, then we have

$$
-\left(\mathcal{L}_{K} z\left(\vartheta_{t} \omega\right), \mathcal{L}_{K} v_{2}\right) \leqslant \frac{1}{6}\left\|\mathcal{L}_{K} v_{2}\right\|^{2}+c\left|y\left(\vartheta_{t} \omega\right)\right|^{2} .
$$

Thus by a combination of (5.55)-(5.58), we find

$$
\begin{aligned}
\frac{d}{d t}\left\|v_{2}\right\|_{X_{0}^{s}}+\left\|\mathcal{L}_{K} v_{2}\right\|^{2} \leqslant & c\left(\|v\|_{2 p-2}^{2 p-2}+\left\|\psi_{3}(t, .)\right\|^{2}+\|g(t, .)\|^{2}\right) \\
& +c\left(\left|y\left(\vartheta_{t} \omega\right)\right|^{2}+\left|y\left(\vartheta_{t} \omega\right)\right|^{2 p-2}\right),
\end{aligned}
$$

where $c>0$ is a deterministic constant independent of $k, \tau$ and $\omega$. Note that we have

$$
\left\|\mathcal{L}_{K} v_{2}\right\|^{2}=\sum_{j=k+1}^{\infty}\left(c_{j} \lambda_{j}\right)^{2} \geqslant \lambda_{k+1} \sum_{j=k+1}^{\infty} c_{j}^{2} \lambda_{j}=\lambda_{k+1}\left\|v_{2}\right\|_{X_{0}^{s}}^{2},
$$

since by the orthogonal property of $e_{j}$ in $X_{0}^{s}$ and $\left\|e_{j}\right\|=1$, we have

$$
\begin{aligned}
\left\|v_{2}\right\|_{X_{0}^{s}}^{2} & =\int_{\mathbf{R}^{2 N}}\left|v_{2}(x)-v_{2}(y)\right|^{2} K(x-y) d x d y \\
& =\int_{\mathbf{R}^{2 N}}\left|\sum_{j=k+1}^{\infty} c_{j} e_{j}(x)-\sum_{j=k+1}^{\infty} c_{j} e_{j}(y)\right|^{2} K(x-y) d x d y \\
& =\sum_{j=k+1}^{\infty} c_{j}^{2} \int_{\mathbf{R}^{2 N}}\left|e_{j}(x)-e_{j}(y)\right|^{2} K(x-y) d x d y \\
& =\sum_{j=k+1}^{\infty} c_{j}^{2} \lambda_{j} \quad(\text { by }(2.10)) .
\end{aligned}
$$

Therefore, combine (5.60) with (5.59) to yield

$$
\begin{aligned}
\frac{d}{d t}\left\|v_{2}\right\|_{X_{0}^{s}}+\lambda_{k+1}\left\|v_{2}\right\|_{X_{0}^{s}}^{2} & \leqslant c\left(\|v\|_{2 p-2}^{2 p-2}+\left\|\psi_{3}(t, .)\right\|^{2}+\|g(t, .)\|^{2}\right) \\
& +c\left(\left|y\left(\vartheta_{t} \omega\right)\right|^{2}+\left|y\left(\vartheta_{t} \omega\right)\right|^{2 p-2}\right) .
\end{aligned}
$$

By Lemma 5.1 (i), we have

$$
\begin{aligned}
& \left\|v_{2}\left(\tau, \tau-t, \vartheta_{-\tau} \omega, v_{\tau-t}\right)\right\|_{X_{0}^{s}} \leqslant \int_{\tau-1}^{\tau} e^{\lambda_{k+1}(\xi-\tau)}\left\|v_{2}\left(\xi, \tau-t, \vartheta_{-\tau} \omega, v_{\tau-t}\right)\right\|_{X_{0}^{s}} d \xi \\
& +c \int_{\tau-1}^{\tau} e^{\lambda_{k+1}(\xi-\tau)}\|v(\xi)\|_{2 p-2}^{2 p-2} d \xi+c \int_{\tau-1}^{\tau} e^{\lambda_{k+1}(\xi-\tau)}\left(\left\|\psi_{3}(\xi, .)\right\|^{2}+\|g(\xi, .)\|^{2}\right) d \xi \\
& +c \int_{\tau-1}^{\tau} e^{\lambda_{k+1}(\xi-\tau)}\left(\left|y\left(\vartheta_{\xi-\tau} \omega\right)\right|^{2}+\left|y\left(\vartheta_{\xi-\tau} \omega\right)\right|^{2 p-2}\right) d \xi
\end{aligned}
$$

Noticing that $\left\|v_{2}\right\|_{X_{0}^{s}} \leqslant\|v\|_{X_{0}^{s}}$, by Lemma 5.3 , there exist positive constants $M=$ $M(\tau, \omega)$ and $T_{1}=T_{1}(\tau, \omega, D) \geqslant 3$ such that for all $t \geqslant T_{1}$,

$$
\begin{aligned}
& \int_{\tau-1}^{\tau} e^{\lambda_{k+1}(\xi-\tau)}\left\|v_{2}\left(\xi, \tau-t, \vartheta_{-\tau} \omega, v_{\tau-t}\right)\right\|_{X_{0}^{s}} d \xi \\
& \leqslant M(\tau, \omega) \int_{\tau-1}^{\tau} e^{\lambda_{k+1}(\xi-\tau)} d \xi \leqslant M(\tau, \omega) \lambda_{k+1}^{-1}
\end{aligned}
$$


For the second term on the right hand of (5.62), by a splitting of the state space $\mathcal{O}$, we have

$$
\begin{aligned}
c \int_{\tau-1}^{\tau} e^{\lambda_{k+1}(\xi-\tau)}\|v(\xi)\|_{2 p-2}^{2 p-2} d \xi= & c \int_{\tau-1}^{\tau} e^{\lambda_{k+1}(\xi-\tau)} \int_{\mathcal{O}}|v(\xi)|^{2 p-2} d x d \xi \\
= & \left(\int_{\tau-1}^{\tau} e^{\lambda_{k+1}(\xi-\tau)} \int_{\tilde{\mathcal{O}}}|v(\xi)|^{2 p-2} d x d \xi\right. \\
& \left.+\int_{\tau-1}^{\tau} e^{\lambda_{k+1}(\xi-\tau)} \int_{\mathcal{O} \backslash \tilde{\mathcal{O}}}|v(\xi)|^{2 p-2} d x d \xi\right)
\end{aligned}
$$

where $\tilde{\mathcal{O}}=\left\{x \in \mathcal{O}:\left|v\left(s, \tau-t, \vartheta_{-\tau} \omega, v_{\tau-t}\right)\right| \geqslant \tilde{h}\right\}$. Then by Lemma 5.4 , there exist constants $T=T(\tau, \omega, D, \varepsilon) \geqslant T_{1} \geqslant 3$ and $N_{0}=N_{0}(\tau, \omega, \varepsilon) \in \mathbf{N}$ such that for all $t \geqslant T, k \geqslant N_{0}$ and a large $\tilde{h}$,

$$
c \int_{\tau-1}^{\tau} e^{\lambda_{k+1}(\xi-\tau)} \int_{\tilde{\mathcal{O}}}|v(\xi)|^{2 p-2} d x d \xi \leqslant c \varepsilon
$$

On the other hand, since $\int_{\mathcal{O} \backslash \tilde{\mathcal{O}}}|v(\xi)|^{2 p-2} d x \leqslant \tilde{h}^{2 p-2} \operatorname{mea}(\mathcal{O} \backslash \tilde{\mathcal{O}}) \triangleq c<\infty$, then

$$
c \int_{\tau-1}^{\tau} e^{\lambda_{k+1}(\xi-\tau)} \int_{\mathcal{O} \backslash \tilde{\mathcal{O}}}|v(\xi)|^{2 p-2} d x d \xi \leqslant c \int_{\tau-1}^{\tau} e^{\lambda_{k+1}(\xi-\tau)} d \xi \leqslant c \lambda_{k+1}^{-1} .
$$

Thus incorporation (5.64)-(5.66), we get

$$
c \int_{\tau-1}^{\tau} e^{\lambda_{k+1}(\xi-\tau)}\|v(\xi)\|_{2 p-2}^{2 p-2} d \xi \leqslant c\left(\varepsilon+\lambda_{k+1}^{-1}\right) .
$$

For the third term of (5.62), by a same argument as (5.43) and (5.44), we may show

$$
c \int_{\tau-1}^{\tau} e^{\lambda_{k+1}(\xi-\tau)}\left(\left\|\psi_{3}(\xi, .)\right\|^{2}+\|g(\xi, .)\|^{2}\right) d \xi \leqslant c \varepsilon
$$

for $k$ large enough. For the last term of (5.62), by (3.12) we have

$$
\begin{aligned}
& c \int_{\tau-1}^{\tau} e^{\lambda_{k+1}(\xi-\tau)}\left(\left|y\left(\vartheta_{\xi-\tau} \omega\right)\right|^{2}+\left|y\left(\vartheta_{\xi-\tau} \omega\right)\right|^{2 p-2}\right) d \xi \\
& =c \int_{-1}^{0} e^{\lambda_{k+1} \xi}\left(\left|y\left(\vartheta_{\xi} \omega\right)\right|^{2}+\left|y\left(\vartheta_{\xi} \omega\right)\right|^{2 p-2}\right) d \xi \\
& \leqslant c \varrho(\omega) \int_{-1}^{0} e^{\left(\lambda_{k+1}-\frac{1}{2} \lambda_{1}\right) \xi} d \xi \leqslant c \varrho(\omega)\left(\lambda_{k+1}-\frac{1}{2} \lambda_{1}\right)^{-1} .
\end{aligned}
$$

Since $\lambda_{k+1} \rightarrow \infty$ as $k \rightarrow \infty$, then by a combination of (5.62), (5.63) and (5.67)-(5.69), there exist positive constants $T=T(\tau, \omega, D, \varepsilon) \geqslant 3$ and $N_{0}=N_{0}(\tau, \omega, \varepsilon) \in \mathbf{N}$ such that for all $t \geqslant T$ and $k \geqslant N_{0}$,

$$
\left\|v_{2}\left(\tau, \tau-t, \vartheta_{-\tau} \omega, v_{\tau-t}\right)\right\|_{X_{0}^{s}}^{2} \leqslant c \varepsilon
$$

where $c>0$ is a constant independent of $\varepsilon, \tau$ and $\omega$. Thanks to $u_{2}(\tau)=v_{2}(\tau)+z_{2}(\omega)$, by employing (5.60) and (3.10), in conjunction with (5.70), it follows that

$$
\begin{aligned}
\left\|u_{2}\left(\tau, \tau-t, \vartheta_{-\tau} \omega, u_{\tau-t}\right)\right\|_{X_{0}^{s}}^{2} & \leqslant\left\|v_{2}\left(\tau, \tau-t, \vartheta_{-\tau} \omega, v_{\tau-t}\right)\right\|_{X_{0}^{s}}^{2}+|y(\omega)|^{2}\left\|\phi_{2}\right\|_{X_{0}^{2}}^{2} \\
& \leqslant c \varepsilon+\varrho(\omega)\left\|\mathcal{L}_{K} \phi\right\|^{2} \lambda_{k+1}^{-1} \leqslant c \varepsilon,
\end{aligned}
$$

when $k$ is large enough, which implies the claims in the lemma.

We are now ready to show that the cocycle $\varphi$ satisfies the $\mathfrak{D}$-flattening condition in the Hilbert space $X_{0}^{s}$, which is a uniformly convex topological space. 
Lemma 5.6. Let $s \in(0,1)$ and $N>2 s$. Suppose that (3.4)-(3.8) hold and $\mathfrak{D}$ is defined by (3.17). Then the cocycle $\varphi$ defined by (3.16) satisfies the $\mathfrak{D}$-flattening condition in $X_{0}^{s}$.

Proof. Given $\varepsilon>0$ arbitrary small, Thanks to Lemma 5.5, for every $\tau \in \mathbf{R}$ and $\omega \in \Omega$, there exist positive constants $T_{1}=T_{1}(\tau, \omega, D, \varepsilon) \geqslant 3$ and a large natural number $N_{0}$ such that, for all $t \geqslant T_{1}$,

$$
\left\|\left(I-P_{N_{0}}\right) u\left(\tau, \tau-t, \vartheta_{-\tau} \omega, u_{\tau-t}\right)\right\|_{X_{0}^{s}} \leqslant \varepsilon,
$$

where $P_{N_{0}}$ is defined by (5.50). On the other hand, by Lemma 5.3, there exist positive constants $T_{2}=T_{2}(\tau, \omega, D, \varepsilon) \geqslant T_{1}$ and $M(\tau, \omega)$ such that, for all $t \geqslant T_{2}$,

$$
\left\|u\left(\tau, \tau-t, \vartheta_{-\tau} \omega, u_{\tau-t}\right)\right\|_{X_{0}^{s}} \leqslant M(\tau, \omega) .
$$

Put $X_{1}=\operatorname{span}\left\{e_{1}, e_{2}, \ldots, e_{N_{0}}\right\}$ where $\left\{e_{j}\right\}_{j \in \mathbf{N}}$ is the basis of $X_{0}^{s}$. Then $X_{1}$ has a finite dimension $N_{0}$ and the bounded projector $P_{N_{0}}: X_{0}^{s} \rightarrow X_{1}$. By the definition of $\varphi$ in (3.16), we have $\varphi\left(t, \tau-t, \vartheta_{-t} \omega,.\right)=u\left(\tau, \tau-t, \vartheta_{-\tau} \omega,.\right)$. Therefore in view of (5.71) and (5.72), we get

$\left\|\left(I-P_{N_{0}}\right) \varphi\left(t, \tau-t, \vartheta_{-t} \omega, u_{\tau-t}\right)\right\|_{X_{0}^{s}} \leqslant \varepsilon, \quad\left\|P_{N_{0}} \varphi\left(t, \tau-t, \vartheta_{-t} \omega, u_{\tau-t}\right)\right\|_{X_{0}^{s}} \leqslant M(\tau, \omega)$, for all $t \geqslant T_{2}$. This completes the proof.

5.3. Pullback attractors in $\boldsymbol{X}_{\mathbf{0}}^{\boldsymbol{s}}$. According to Lemma 4.3, Lemma 4.4 and Lemma 5.6, in conjunction with Theorem 2.8 and Theorem 2.9, we now obtain the main result in this section.

Theorem 5.7. Let $s \in(0,1)$ and $N>2 s$. Suppose that (3.4)-(3.8) hold and $\mathfrak{D}$ is defined by (3.17). Then the random cocycle $\varphi$ defined by (3.16) admits a unique D-pullback attractor $\mathcal{A}_{X_{0}^{s}}=\left\{\mathcal{A}_{X_{0}^{s}}(\tau, \omega): \tau \in \mathbf{R}, \omega \in \Omega\right\}$ in $X_{0}^{s}$, where $\mathcal{A}_{X_{0}^{s}}(\tau, \omega)$ is structured by

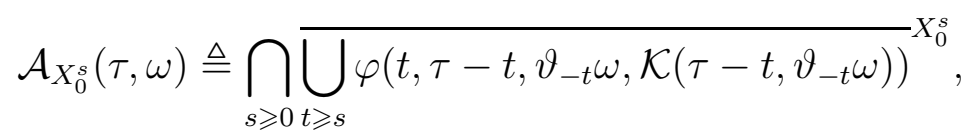

where the family $\mathcal{K}$ is the closed $\mathfrak{D}$-pullback random absorbing of $\varphi$ in $L^{2}(\mathcal{O})$. In addition, $\mathcal{A}_{X_{0}^{s}}=\mathcal{A}$, where $\mathcal{A}$ is the $\mathfrak{D}$-pullback attractor of $\varphi$ in $L^{2}(\mathcal{O})$ as in Theorem 4.5.

Remark 5. Theorem 5.7 demonstrates that the general model (1.1)-(1.3) admits the smoothing dynamics, namely, the compactness and attracting properties of pullback attractor obtained in $L^{2}(\mathcal{O})$ can be generalized to the regular space $X_{0}^{s}$, despite of the random influence. To achieve this regular dynamics, it seems that the noise coefficient $\phi \in L^{\infty}$ is indispensable, which is intrinsically used in Lemma 5.4. In recent literature $[32,30,33]$, the author developed a method to surmount the condition $\phi \in L^{\infty}$. We will discuss the pullback attractor for this equation on unbounded domain driven by such type noise in the future.

Acknowledgments. This work was supported by CTBU Grant 1751041, China NSF Grant 11601046 and Chongqing key laboratory of social economy and applied statistics. 


\section{References}

[1] Arnold, L.: Random dynamical system. - Springer-Verlag, Berlin, 1998.

[2] BARtsch, T., and Z. Liv: On a supperlinear elliptic $p$-Laplacian equation. - J. Differential Equations 198, 2004, 149-179.

[3] Chueshov, I.: Monotone random systems theory and applications. - Springer-Verlag, Berlin, 2002.

[4] Crauel, H., A. Debussche, and F. Flandoli: Random attractors. - J. Dynam. Differential Equations 9, 1997, 307-341.

[5] Crauel, H., and F. Flandoli: Attracors for random dynamical systems. - Probab. Theory Related Fields 100, 1994, 365-393.

[6] Crauel, H., and P.E. Kloeden: Nonautonomous and random attractors. - Jahresber. Deutsch. Math.-Verein. 117, 2015, 173-206.

[7] Di Nezza, E., G. Palatucci, and E. Valdinoci: Hitchhikers guide to the fractional Sobolev spaces. - Bull. Sci. Math. 136, 2012, 521-573.

[8] Dipierro, S., S. PATrizi, and E. VAldinoci: Chaotic orbits for systems of nonlocal equations. - Comm. Math. Phys. 349, 2017, 583-626.

[9] Ferrara, M., G. Molica Bisci, and B. Zhang: Existence of weak solutions for non-local fractional problems via Morse theory. - Discrete Contin. Dyn. Syst. Ser. B 19, 2014, 2483-2499.

[10] Fiscella, A., R. Servadei, and E. Valdinoci: Density properties for fractional Sobolev spaces. - Ann. Acad. Sci. Fenn. Math. 40, 2015, 235-253.

[11] Flandoli, F., and B. Schmalfuss: Random attractors for the 3D stochastic Navier-Stokes equation with multiplicative noise. - Stoch. Stoch. Rep. 59, 1996, 21-45.

[12] Giacomoni, J., P. K. Mishra, and K. Sreenadh: Fractional elliptic equations with critical exponential nonlinearity. - Adv. Nonlinear Anal. 5, 2016, 57-74.

[13] Gu, A., D. LI, B. WANG, and H. YAng: Regularity of random attractors for fractional stochastic reaction-diffusion equations on $R^{n}$. - J. Differential Equations 264, 2018, 7094-7137.

[14] Kloeden, P.E., and J. A. Langa: Flattening, squeezing and the existence of random attractors. - Proc. R. Soc. Lond. Ser. A 463, 2007, 163-181.

[15] Kloeden, P. E., and T. Lorenz: Construction of nonautonomous forward attractors. - Proc. Amer. Math. Soc. 144, 2016, 259-268.

[16] Kloeden, P. E., and M. Yang: Forward attraction in nonautonomous difference equations. - J. Difference Equ. Appl. 22, 2016, 1027-1039.

[17] Lu, H., P. W. Bates, J. Xin, and M. Zhang: Asymptotic behavior of stochastic fractional power dissipative equations on $R^{n}$.- Nonlinear Anal. 128, 2015, 176-198.

[18] Lu, H., P. W. Bates, S. Lu, and M. Zhang: Dynamics of 3D fractional complex GinzburgLandau equation. - J. Differential Equations 259, 2015, 5276-5301.

[19] Lu, H., P. W. Bates, S. Lu, and M. Zhang: Dynamics of the 3D fractional GinzburgLandau equation with multiplicative noise on an unbounded domain. - Commun. Math. Sci. 14, 2016, 273-295.

[20] Molica Bisci, G., V.D. RĂdulescu, and R. Servadei: Variational methods for nonlocal fractional problems. - Encyclopedia Math. Appl. 162, Cambridge Univ. Press, Cambridge, 2016.

[21] Molica Bisci, G., and D. Repovš: Existence and localization of solutions for nonlocal fractional equations. - Asymptotic Anal. 90, 2014, 367-378.

[22] Molica Bisci, G., and R. Servadei: A Brezis-Nirenberg splitting approach for nonlocal fractional equations. - Nonlinear Anal. 119, 2015, 341-353. 
[23] Schmalfuss, B.: Backward cocycle and attractors of stochastic differential equations. - In: International Seminar on Applied Mathematics-Nonlinear Dynamics: Attractor Approximation and Global Behavior, edited by V. Reitmann, T. Riedrich, N. Koksch, Technische Universität, Dresden, 1992, 185-192.

[24] Servadei, R., and E. VAldinoci: Mountain pass solutions for non-local elliptic operators. J. Math. Anal. Appl. 389, 2012, 887-898.

[25] Servadei, R., and E. VAldinoci: Variational methods for non-local operators of elliptic type. - Discrete Contin. Dyn. Syst. 33, 2013, 2105-2137.

[26] Servadei, R., and E. VAldinoci: The Brezis-Nirenberg result for the fractional Laplacian. Trans. Amer. Math. Soc. 367, 2015, 67-102.

[27] WANG, B.: Suffcient and necessary criteria for existence of pullback attractors for non-compact random dynamical systems. - J. Differential Equations 253, 2012, 1544-1583.

[28] WANG, B.: Random attractors for non-autonomous stochastic wave euqations with multiplicative noises. - Discrete Contin. Dyn. Syst. 34, 2014, 269-330.

[29] WANG, B.: Asymptotic behavior of non-autonomous fractional stochastic reaction-diffusion equations. - Nonlinear Anal. 158, 2017, 60-82.

[30] Zhно, W.: Random dynamics of stochastic $p$-Laplacian equations on $\mathbf{R}^{N}$ with an unbounded additive noise. - J. Math. Anal. Appl. 455, 2017, 1178-1203.

[31] Zhно, W.: Long-time random dynamics of stochastic parabolic $p$-Laplacian equations on $\mathbf{R}^{N}$. - Nonliner Anal. 152, 2017, 196-219.

[32] Zhaо, W.: Continuity and random dynamics of the non-autonomous stochastic FitzHughNagumo system on $\mathbf{R}^{N}$. - Comput. Math. Appl. 75, 2018, 3801-3824.

[33] Zhaо, W.: Random dynamics of non-autonomous semi-linear degenerate parabolic equations on $\mathbf{R}^{N}$ driven by an unbounded additive noise. - Discrete Contin. Dyn. Syst. Ser. B 23, 2018, 2499-2526.

[34] Zhaо, W., and Y. Zhang: Compactness and attracting of random attractors for nonautonomous stochastic lattice dynamical systems in weighted space $\ell_{\rho}^{p}$. Appl. Math. Comput. $291,2016,226-243$.

[35] Zhou, Q., and K. WANG: Existence and multiplicity of solutions for nonlinear elliptic problems with the fractional Laplacian. - Fract. Calc. Appl. Anal. 18, 2015, 133-145.

Received 18 May $2018 \bullet$ Revision received 10 August $2018 \bullet$ Accepted 13 August 2018 